\title{
Constitutive theories for woven composite structures subjected to shock loading; experimental validation using a conical shock tube
}

\author{
David R. Hufner* \\ General Dynamics Electric Boat, Groton, CT, USA
}

Received 4 March 2010

Revised 7 October 2010

\begin{abstract}
Woven polymer-based composites are currently used in a wide range of marine applications. These materials often exhibit highly nonlinear, rate dependent, anisotropic behavior under shock loadings. Correlation to transient response data, beyond an initial peak, is often difficult. The state of damage evolves throughout the time history and the unloading response varies based on the amount, and nature of, the accumulated damage. Constitutive theories that address the loading and unloading responses have been developed and integrated with each other. A complete theory, applicable to transient dynamic analysis, is presented. The model is implemented within the commercial finite element code, Abaqus, in the form of a user material subroutine. In this study, the conical shock tube is used to experimentally reproduce the high strain rates and fluid structure interactions typical of underwater shock loadings. The conical shock tube data is used to validate analytical model predictions. Simulation results are in good agreement with test data.
\end{abstract}

\section{Introduction}

The designs of many composite components are controlled by transient dynamic (shock) loadings. Compared to normal operating loads, these events are typically severe, and involve much higher loads and pressures. It is no surprise that linear analysis techniques, coupled with standard interactive failure criterion (e.g., Tsai-Wu, Tsai-Hill), predict lamina level (ply) failures for significant portions of the structure. Due to the uncertainty in predicting the nonlinear response of composites, a common design practice is to require that the composite remain linear (and undamaged) under even the most severe design loads. Attempting to design composite components using a strictly linear response criterion often results in thicker, heavier, more expensive designs that do not take full advantage of the benefits of composites. Woven composites often have high toughness, damage tolerance, and considerable durability. The ability of composite materials to perform well after the onset of damage has been demonstrated experimentally, both statically and dynamically [1-3]. However, the mechanical behavior beyond first ply failure is difficult to characterize due to material nonlinearity, strain rate dependence, and progressive failure. The ability to predict non-linear rate dependent response and characterize damage can lead to more potential composite applications and more optimized designs.

\footnotetext{
*Address for correspondence: General Dynamics Electric Boat, 75 Eastern Point Road, Groton, CT, USA. Tel.: +1 860433 3354; E-mail: dhufner@gdeb.com.
} 
The use of advanced woven composite material models for transient analyses requires a considerable test and validation effort. This is often the case since linear models already provide a high enough level of confidence (when stresses are limited to values within the linear regime) for many design cases. Application of advanced non-linear theories to transient dynamics must demonstrate some added benefit in order to be considered worthwhile (e.g., inclusion of material non-linearity, strain rate dependence, and progressive failure). When these phenomena are significant, special attention must be paid to modeling the unloading response. Material unloading can be expected for a significant portion of many transient analyses, and will be influenced by the damage level and type of damage present.

In general terms, advanced constitutive theories for composites have utilized two uniquely different forms. A common approach involves defining non-linear constitutive theory, in which nonlinearity is represented through an 'effective' stress and plastic strain. This approach is similar to classical plasticity theory in metals. Familiar concepts include the use of a plastic potential function, an associated flow rule, the equivalence of plastic work rates, and the plastic modulus. Recently, several investigators have developed plastic potential functions that are suitable for use with woven composites [4,5].

Another popular approach involves application of continuum damage theory [6-11]. Continuum damage mechanics is based on the concept that load can only be resisted by the undamaged area. The stress acting on the undamaged area is proportionately higher and can be related to the stress on the total area through a set of internal state variables known as damage parameters. Each mode of damage (e.g., fiber tension, fiber compression, matrix shear) is tracked by a separate damage parameter, allowing a fully anisotropic characterization of composite damage. The rate of change of damage parameters (a.k.a. damage evolution laws) is normally derived from the energy or thermodynamic forces associated with the failure process.

An obvious advantage to defining non-linear constitutive theory is that sub-critical damage (resulting from matrix micro-cracking or interfacial bond failures) is already accounted for in the non-linear characterization. However, most theories formulated in this manner address monotonic loading, and do not specifically address the unloading response that would be expected for transient responses. Consequently, unloading would occur in non-physical fashion by re-tracing the same path experienced during the 'loading' response. Continuum damage theories provide a better framework to characterize unloading response since constitutive behavior is characterized by a damaged elasticity matrix. As such, the unloading response is always influenced by the load level and damage state. In this paper a new concept is presented in which non-linear constitutive theory is used to represent sub-critical damage development, but concepts from continuum damage mechanics are incorporated to characterize unloading from various load levels that may involve different states of damage. The combination of these theories is specifically intended for transient analysis of woven composite structures subjected to shock loads, but can also be applied to a wide range of monotonic or transient loadings.

Validated dynamic material characterizations are essential to expand the use of composite materials in shock environments. This often begins with small-scale laboratory experiments that involve low strain rates, in-plane multiaxial loadings, and no consideration of fluid-structure interaction. Although good correlation may be achieved in these tests, use of advanced material models in design applications requires a higher level of validation. The ability to predict the response of simple structures under representative loadings must also be demonstrated. These tests require unique experimental methods that can represent the basic nature of an underwater shock loading. Specifically, experiments must reproduce strain rate magnitudes corresponding to pressure waves traveling at the speed of sound through an acoustic fluid media. The conical shock tube is one such test method that is able to achieve this.

The conical shock tube tests used in this study were performed in 2004 by Baker and Telegadas [12]. This paper describes a recent simulation effort, which used the conical shock tube data to evaluate a new composite material model. Finite element models of the conical shock tube experiment (including the specimen, test fixture, and a portion of the fluid) are developed in Abaqus. Dynamic fluid-structure interaction simulations are conducted using the explicit dynamics solution procedure. Simulation results are compared with test data. The objective of this work is to develop more robust modeling techniques to simulate the behavior of composites for high-rate underwater shock loading. This involves consideration of material non-linearity, strain rate dependence, and progressive failure. More robust modeling techniques, combined with validated dynamic material characterizations, will allow the use of composite materials in a wider range of potential applications. 


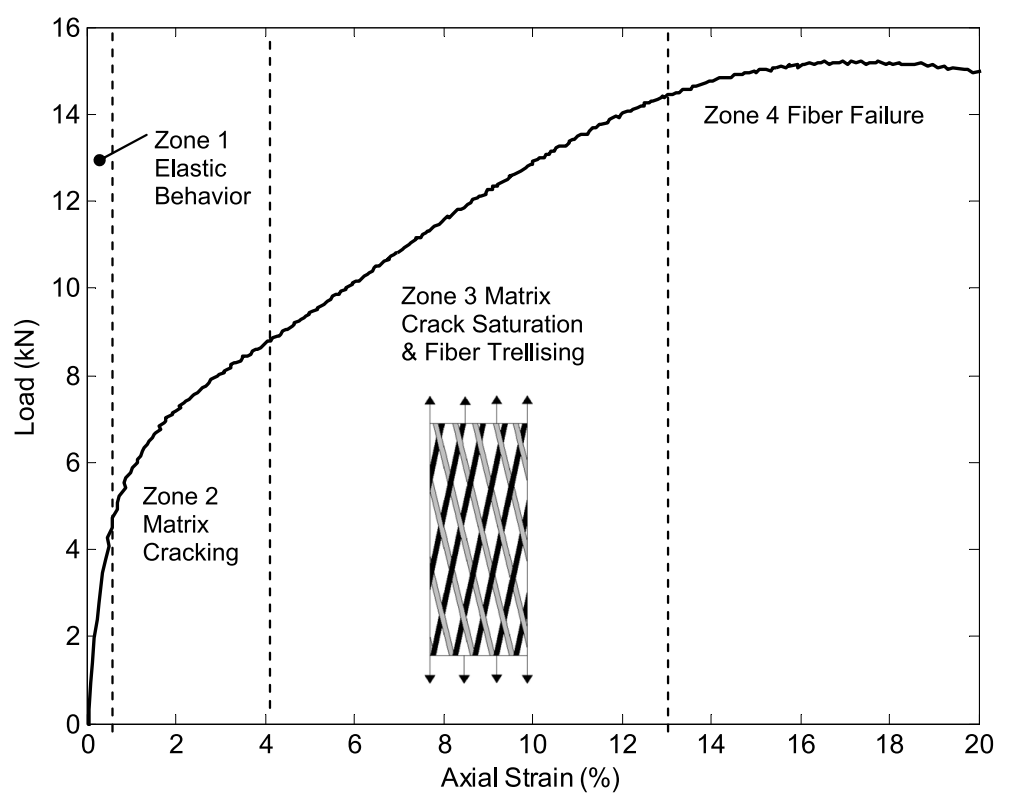

Fig. 1. Typical off-axis response of woven composite.

\section{Theory}

To develop a constitutive theory that is appropriate for a woven polymer based composite, we must first consider the basic nature of the response. Although behavior in the fiber directions can often be approximated as linear, the response for off-axis load orientations exhibits significant non-linearity and very high strain to failure. A sample plot showing the response of a $45^{\circ}$ tension specimen is shown in Fig. 1. In this test, the load is oriented at a $45^{\circ}$ angle with respect to the material reinforcement directions (i.e., weave directions). For discussion, the response can be separated into four zones, which each show a different type of behavior. As loading begins, an initial elastic response is observed (zone 1). The behavior is mostly elastic up to approximately $0.5 \%$ strain. At this point, matrix cracking begins to occur (zone 2). As the load increases, the density of the matrix cracks keeps increasing, and the response becomes non-linear. At about $4 \%$ strain (zone 3 ), the density of matrix cracks reaches a saturation level, and very few new cracks are formed. The concept of crack saturation, also known as the 'characteristic damage state,' is a natural phenomena that occurs in many physical systems such as stratified rock or highway surfaces [13]. Once a state of crack saturation is reached, the non-linearity resulting from matrix cracking is no longer prevalent. In this zone, the behavior is primarily controlled by the fibers. The fibers have a tendency to re-orient themselves towards the direction of the loading vector. This behavior is referred to as trellising, and is said to occur whenever the angle between reinforcement directions changes from $90^{\circ}$. Fiber trellising continues until about $13 \%$ strain, where the fibers eventually begin to fail (zone 4). The final non-linearity is the likely result of statistically based fiber failure over a range of axial strain.

The behavior for zones 1 and 2 occurs at relatively small strains and is suitable for characterization with a nonlinear constitutive theory. A non-linear, rate dependent, constitutive theory has been developed based on the plastic potential function proposed by Ogihara and Reifsnider [5] for woven composites. This potential function consists of 4 terms and is based on Hill's generalized plastic potential for a 3D orthotropic solid modified for 2D plane stress behavior in an individual lamina.

$$
2 f\left(\sigma_{i j}\right)=a_{11} \sigma_{11}^{2}+a_{22} \sigma_{22}^{2}+2 a_{12} \sigma_{11} \sigma_{22}+2 a_{66} \sigma_{12}^{2}
$$

Ogihara and Reifsnider determined an experimental procedure to determine each of the four unknown potential function parameters $\left(a_{11}, a_{12}, a_{22}\right.$, and $\left.a_{66}\right)$. This procedure involved performing basic tension tests with various fiber orientations. After plotting the effective stress vs effective plastic strain curve for each test, the correct combination of potential function parameters results in all curves collapsing to a single "master" curve. The non-linear material 
behavior could then be characterized by the master curve. A power law fit was initially used to define the master curve [14].

$$
\bar{\varepsilon}^{p}=A \bar{\sigma}^{n}
$$

Weeks and Sun [15] determined that the same exponent $n$ could be applied to all strain rates. Therefore, the rate dependence could be characterized within a single parameter, $A$. Repeating the experimental procedure at various strain rates permits characterization of rate dependence using a form first proposed by Thiruppukuzhi and Sun [16]:

$$
\bar{\varepsilon}^{p}=\chi\left(\overline{\dot{\varepsilon}}^{p}\right)^{m} \bar{\sigma}^{n}
$$

This scalar equation relates effective stress and effective plastic strain rate to the effective plastic strain for arbitrary cases of multi-axial stress. Differentiation results in a visco-plastic modulus $\left(H_{p}\right)$ that can be incorporated into a continuum theory.

$$
H_{p}=\frac{d \bar{\sigma}}{d \bar{\varepsilon}^{p}}=\frac{\dot{\bar{\sigma}}}{\overline{\dot{\varepsilon}}^{p}}=\frac{1}{n \chi\left(\overline{\dot{\varepsilon}}^{p}\right)^{m} \bar{\sigma}^{n-1}}
$$

Using these concepts, the author has developed a complete set of non-linear anisotropic constitutive relations that could be applied to multi-axial non-uniform stress states [17,18]. Strain rate effects are included in both linear and non-linear responses, as well as through definition of rate dependent failure functions. Progressive failure is introduced through a strain/deformation based failure criterion that is based on the strain in the rotated fiber direction. After failure is reached the remaining stiffnesses at the failed material point are decreased to negligible levels using exponential degradation function. Other unique phenomena, such as matrix crack saturation and changes in on-axis non-linear response due to weave effects, are also addressed. These aspects are explained in detail by prior works [17, 18]. The complete progressive failure approach is implemented within the Abaqus finite element code with a user material subroutine (UMAT, VUMAT).

Initially, laboratory testing of open hole tension (OHT) specimens, using digital image correlation, was performed to validate the theory and corresponding finite element implementation. Results comparisons are shown in Fig. 2 through Fig. 5. The OHT specimens are cut from a 10 ply woven E-glass / Vinylester panel with a warps parallel lay-up. Specimens are cut at $0^{\circ}$ and $45^{\circ}$ orientations (i.e., angle between warp fibers and loading direction).

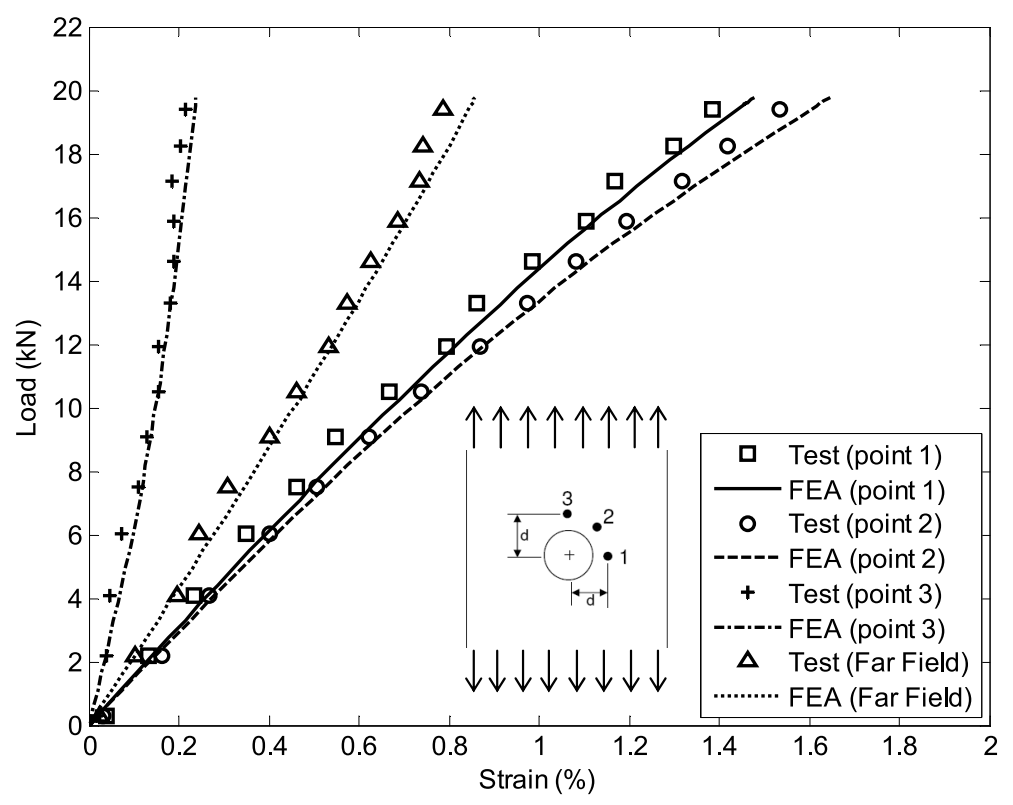

Fig. 2. $0^{\circ}$ open hole tension, comparison of simulation and test. 


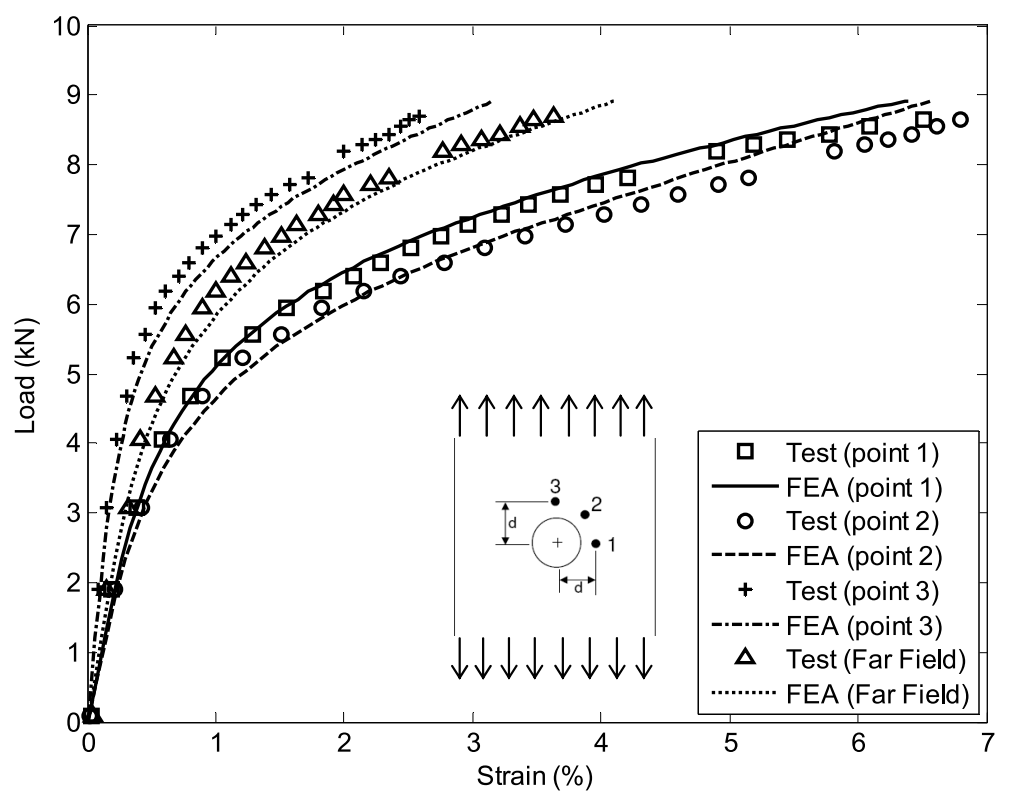

Fig. 3. $45^{\circ}$ open hole tension, comparison of simulation and test.
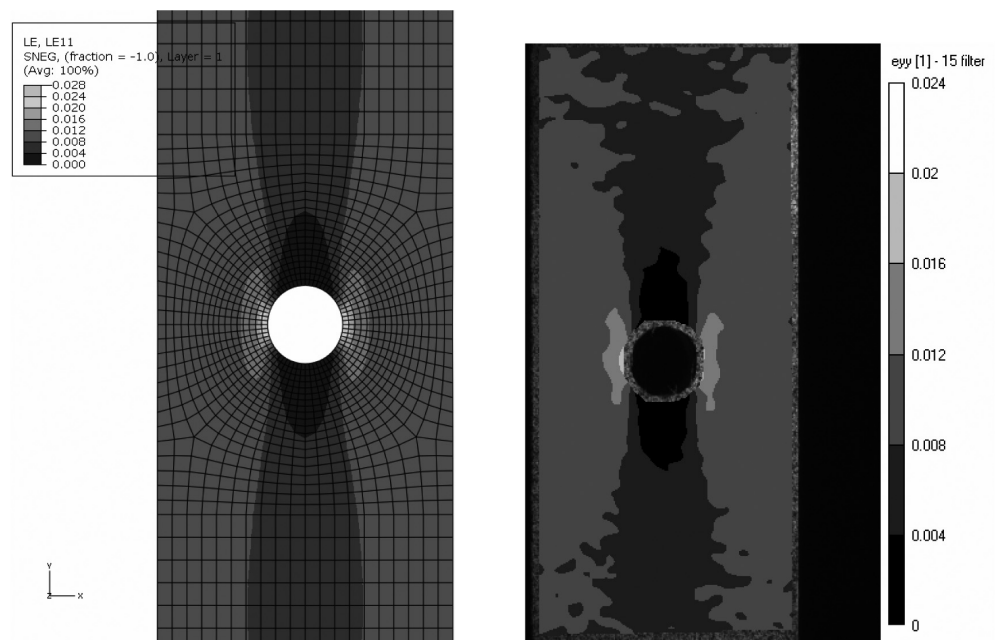

Fig. 4. $0^{\circ}$ OHT longitudinal strain contours from simulation and test, $15.6 \mathrm{kN}(3500 \mathrm{lbs})$.

Although good correlation is seen between analysis and test, if unloading were to occur the model would predict a complete re-tracement all the way back to the original configuration. This behavior is not physically possible. The preferred approach is to model a linear unloading stiffness that can change based on the level of damage and the type of damage. This has lead to further enhancements to specifically address the unloading response.

To define a constitutive theory for the unloading response, it is first necessary to develop a criterion for "loading". The loading criterion is based on a characteristic loading function $g_{i}$ and an accumulation threshold $r_{i}$. The characteristic loading function describes the magnitude of the loading at the current time, while the accumulation threshold is the maximum value of $g_{i}$ between $t_{0}$ and the current time. Thus, a negative (positive) value of $f_{i}$ corresponds to unloading (loading).

$$
f_{i}=g_{i}-r_{i}
$$




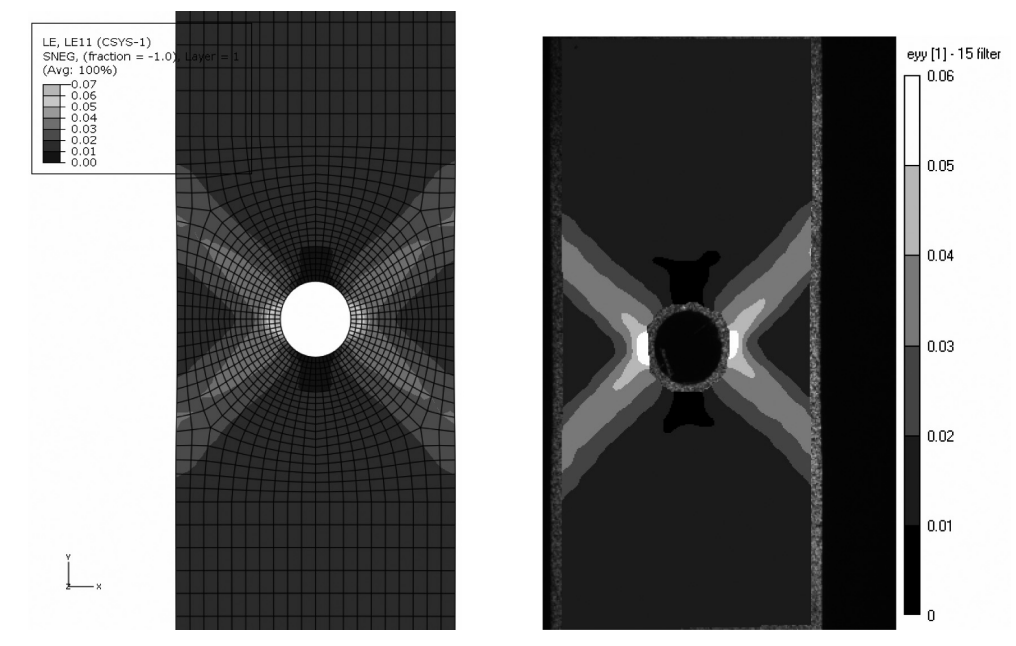

Fig. 5. $45^{\circ}$ OHT, longitudinal strain contours from simulation and test, $6.7 \mathrm{kN}(1500 \mathrm{lbs})$.

Note that the above criterion is also satisfied for cases involving re-loading. Re-loading is also interpreted as 'unloading' since the loading function has not surpassed a maximum value achieved in a previous step. This should not result in an increase in damage. Only 'loading' as defined by Eq. (5) produces an increase in damage.

Characteristic loading function(s) can take various forms. In continuum plasticity, a yield function is defined and checks are performed at each time step to determine if the stress increment is outside the yield surface. An analogous approach for the woven composite is to use the value of the plastic potential given by Eq. (1). Optionally, the trace of the stress tensor $\left(\sigma_{i i}\right)$ could be used to track separate $g_{i}$ for tension and compression. This approach can be useful for cases where a separate set of non-linear parameters is determined from compression tests.

$$
g_{i}=\frac{1}{2}\left[a_{11} \sigma_{11}^{2}+a_{22} \sigma_{22}^{2}+2 a_{12} \sigma_{11} \sigma_{22}+2 a_{66} \sigma_{12}^{2}\right]
$$

On the other hand, continuum damage theories define loading or unloading based on the values of the failure functions. Hence, $g_{i}$ may be taken as the failure function value for each mode. In this fashion, loading is based on advancing the progress toward failure.

$$
g_{i}=F a_{i}
$$

To date, both definitions have been successfully implemented. However, more stable computational performance is observed using the plastic potential as the characteristic loading function.

An orthotropic elastic response is assumed to characterize the unloading constitutive behavior. The unloading stiffness is controlled by internal damage parameters, $\omega$, which are defined separately for warp tension, fill tension, and shear. A damage parameter equal to 0 corresponds to the undamaged lamina, while a damage parameter of 1 implies full damage (failure). In our case, the physical meaning of the damage parameters is consistent with established continuum damage theories (e.g., [6-11]), which utilize internal state variables to track damage evolution in each mode. The damage parameters represent the loss of area associated with resisting each type of loading. The loss of area produces a higher effective stress since only the undamaged area is assumed to resist the load. The effective stress $\hat{\sigma}$ acting on the undamaged area is related to the nominal (true) stress by the damage operator tensor, M.

$$
\hat{\sigma}=\mathrm{M} \sigma
$$

where,

$$
\boldsymbol{\omega}=\left\{\begin{array}{l}
\omega_{11} \\
\omega_{22} \\
\omega_{12}
\end{array}\right\} \quad \hat{\boldsymbol{\sigma}}=\left\{\begin{array}{l}
\hat{\sigma}_{11} \\
\hat{\sigma}_{22} \\
\hat{\sigma}_{12}
\end{array}\right\} \quad \boldsymbol{\sigma}=\left\{\begin{array}{l}
\sigma_{11} \\
\sigma_{22} \\
\sigma_{12}
\end{array}\right\} \quad \mathbf{M}=\left[\begin{array}{ccc}
\frac{1}{\left(1-\omega_{11}\right)} & 0 & 0 \\
0 & \frac{1}{\left(1-\omega_{22}\right)} & 0 \\
0 & 0 & \frac{1}{\left(1-\omega_{12}\right)}
\end{array}\right]
$$


The effective stress is then used in the compliance relation, in place of the nominal stress.

$$
\varepsilon=\mathbf{C} \hat{\boldsymbol{\sigma}}=\mathbf{C M} \boldsymbol{\sigma}=\mathbf{C}^{\omega} \boldsymbol{\sigma}
$$

So that the compliance of the damaged lamina $\mathbf{C}^{\omega}$ is defined as:

$$
\mathbf{C}^{\omega}=\left[\begin{array}{ccc}
\frac{1}{\left(1-\omega_{11}\right) E_{1}} & -\frac{\nu_{12}}{E_{1}} & 0 \\
-\frac{\nu_{12}}{E_{1}} & \frac{1}{\left(1-\omega_{22}\right) E_{2}} & 0 \\
0 & 0 & \frac{1}{\left(1-\omega_{12}\right) G_{12}}
\end{array}\right]
$$

Here we use a simplified version of the damaged compliance matrix. Based on rationale from Matzenmiller et al. the effect of the damage in the 1-2 and 2-1 terms is neglected. As long as the damage parameters are less than 1, the inverse of $\mathbf{C}^{\omega}$ exists. The stiffness of the damaged lamina $\mathbf{Q}^{\omega}$ is:

$$
\mathbf{Q}^{\omega}=\frac{1}{D}\left[\begin{array}{ccc}
\left(1-\omega_{11}\right) E_{1} & \left(1-\omega_{11}\right)\left(1-\omega_{22}\right) \nu_{21} E_{1} & 0 \\
\left(1-\omega_{11}\right)\left(1-\omega_{22}\right) \nu_{12} E_{2} & \left(1-\omega_{22}\right) E_{2} & 0 \\
0 & 0 & D\left(1-\omega_{12}\right) G_{12}
\end{array}\right]
$$

With

$$
D=1-\left(1-\omega_{11}\right)\left(1-\omega_{22}\right) \nu_{21} \nu_{12}, \text { and } \nu_{21}=\nu_{12} \frac{E_{2}}{E_{1}}
$$

In continuum damage theories, determination of damage parameters $\boldsymbol{\omega}$ would normally require the derivation of evolution equations that describe the time rate of change of $\boldsymbol{\omega}$ with respect to the rate of change of stress or strain components. This derivation can take various forms. Although common approaches require some definition of fracture energy as the initial material property input. In this case, since a nonlinear constitutive model is used, the level of damage can be inferred from the non-linear stresses and strains. This leads to a unique procedure for determination of the damage parameters.

Damage parameters are determined based on recoverable energy. As shown in Fig. 6, the maximum recoverable energy is the energy that would be recovered if the entire load were removed and the state of deformation returns to the original configuration. An unloading from point A corresponds to a maximum recoverable energy equal to the area of triangle $\mathrm{OAB}$. If we proceed with the assumption that the material unloads and returns to its original configuration (point $\mathrm{O}$ ), we may associate the level of damage with maximum recoverable energy.

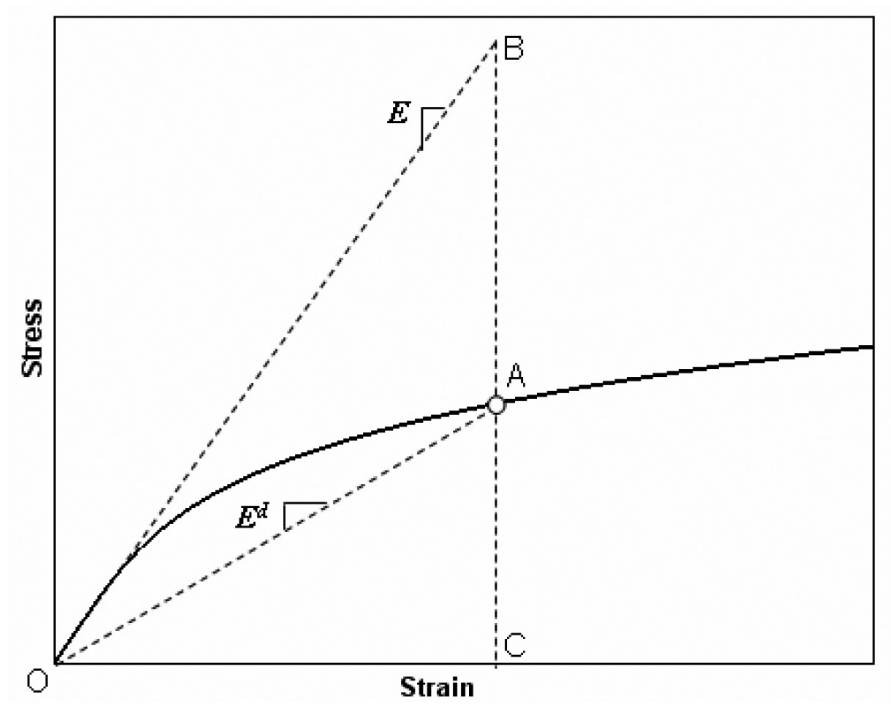

Fig. 6. Determination of damage from non-linear stress-strain response.

To begin, we recall from classical continuum damage mechanics [6] that a "continuity" parameter $\psi$ is defined such that the effective stress (acting on the undamaged area) can be related to the true stress (acting on total area). 


$$
\hat{\sigma}=\frac{\sigma}{\psi}
$$

The continuity is a positive monotonically decreasing scalar variable that is equal to 1 for the undamaged state and decreases to zero as damage accumulates. Assuming linear elasticity, this implies that the damaged elastic modulus is related to the virgin modulus by the continuity parameter.

$$
E^{d}=\psi E
$$

As shown in Fig. 6, both the damaged modulus and virgin modulus can be determined graphically from the non-linear stress-strain curve. Therefore, the continuity parameter will be the same as the ratio of strain energy densities.

$$
\psi=\frac{\text { Maximum Recoverable Energy }}{\text { Psuedo }- \text { Elastic Energy }}=\frac{\text { Area OAC }}{\text { Area OBC }}
$$

In modern continuum damage theories, the continuity parameter is replaced by the damage parameter $\omega$, which is related to continuity by $\psi=1-\omega$. That is, the definition of damage is reversed such that the internal state variable equals zero (instead of 1 ) for the undamaged state. Substitution and rearranging the above equations results in the damage parameter for this simple 1D case.

$$
\omega=1-\frac{\text { Area OAC }}{\text { Area } \mathrm{OBC}}=1-\frac{\sigma_{A}}{E \varepsilon_{A}}
$$

Thus far, it has been assumed that the maximum amount of energy density can be recovered upon unloading, which means that the material unloads back to its original configuration. Of course, this is an idealized scenario and real composites are unlikely to behave this way. However, a secant unloading back to nearly the original shape is common. The elastic action of the fibers pulls the material back together and causes open matrix cracks to close. The elastic (linear) strain is entirely recovered, plus some unknown portion of the plastic (nonlinear) strain. Although it is possible to characterize the unloading with sufficient testing, this data is often unavailable. For this reason, we have formulated a theory that allows specification of a single parameter that controls unloading. This provides the ability to bound specific transient problems by selecting different values for the unloading parameter.

We introduce a new material parameter known as the plastic recovery factor $\xi$. The plastic recovery factor represents the ratio of the plastic (nonlinear) strain that would be recovered with complete load removal. As shown in Fig. 7, a plastic recovery factor of 1.0 implies that all plastic strain is recovered and unloading occurs along a line segment directed at the origin. A plastic recovery factor of 0 implies that only elastic strain is recovered and unloading occurs along a line segment parallel to the initial elastic response.

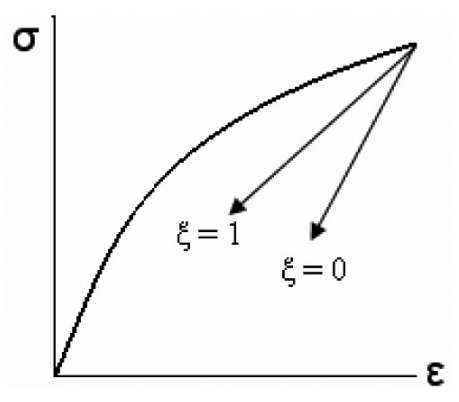

Fig. 7. Plastic recovery factor.

The $\xi$ parameter chosen defines the basic characteristics and assumptions regarding material unloading. To account for various $\xi$ values, the damage parameters $\left(\omega_{11}, \omega_{22}\right.$, and $\left.\omega_{12}\right)$ require adjustment. This graphical procedure is illustrated in Fig. 8. Assuming an arbitrary plastic recovery factor between 0 and 1 , segment AE is shifted horizontally to the left such that point $E$ is coincident with point $O$ and point A moves to A'. Segment OA' (now parallel to AE) is then extended such that it intersects segment AB. The damage parameter is then determined based on an 'adjusted' recoverable energy that is defined by the area of triangle OFC. The unloading response for an arbitrary $\xi$ is governed by the damage parameter: 


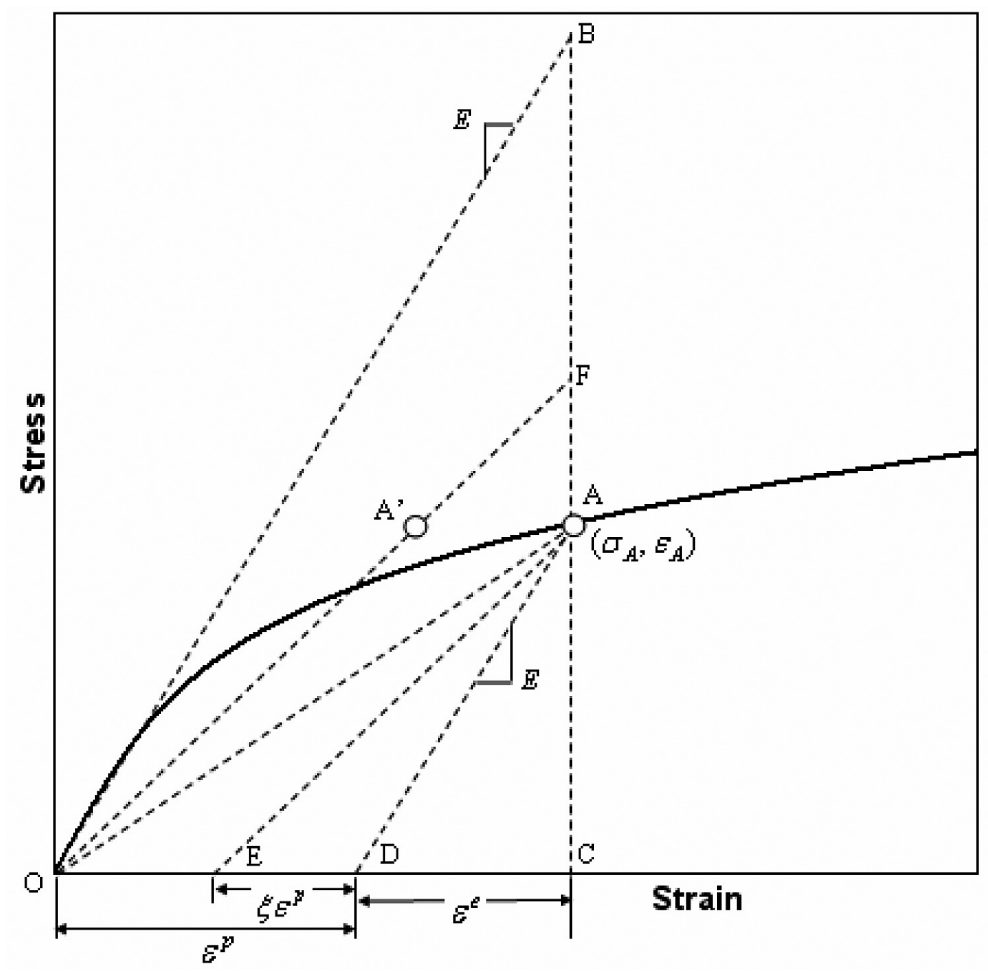

Fig. 8. Graphical determination of damage parameter, $\omega$.

$$
\omega=1-\frac{\text { Area OFC }}{\text { Area OBC }}=\frac{\xi\left(E \varepsilon_{A}-\sigma_{A}\right)}{\sigma_{A}+\xi\left(E \varepsilon_{A}-\sigma_{A}\right)}
$$

In Eq. (16), we note that $\xi=1$ produces the same result as Eq. (15), and $\xi=0$ results in a damage parameter of zero. If $\omega=0$, Eq. (11) reduces to the undamaged orthotropic elastic stiffness and unloading will resemble metal plasticity (segment AD).

The damage parameters $\omega$ are recalculated at every time increment where the loading criterion of Eq. (5) is satisfied. When unloading or reloading is taking place, $\omega$ is held constant since it is assumed that no additional damage accumulation occurs during unloading or reloading time steps. A generalized description of the damage parameters, applicable to step-wise analysis of multi-axial dynamic loading, is given by:

$$
\begin{aligned}
& \omega_{i j}^{k}=\frac{\xi\left(\bar{E} \bar{\varepsilon}_{i j}^{k}-\sigma_{i j}^{k}\right)}{\sigma_{i j}^{k}+\xi\left(\bar{E} \bar{\varepsilon}_{i j}^{k}-\sigma_{i j}^{k}\right)} \text { If } f_{i}^{k} \geqslant 0 \\
& \omega_{i j}^{k}=\omega_{i j}^{k-1} \text { If } f_{i}^{k}<0
\end{aligned}
$$

where $k$ is the time step number, and the effective modulus $\bar{E}$ is defined accordingly for each damage parameter equation.

$$
\begin{aligned}
& \omega_{11} \text { Equation }: \bar{E}=\frac{E_{11}}{1-\nu_{12} \nu_{21}} \\
& \omega_{22} \text { Equation }: \bar{E}=\frac{E_{22}}{1-\nu_{12} \nu_{21}} \\
& \omega_{12} \text { Equation }: \bar{E}=G_{12}
\end{aligned}
$$

To implement the graphical procedure for determination of damage parameters $\omega_{11}$ and $\omega_{22}$, it is necessary to define a single strain value conjugate to each of the normal stresses. Hence, modified strain values $\left(\bar{\varepsilon}_{11}\right.$ and $\left.\bar{\varepsilon}_{22}\right)$ are 
introduced in Eq. (17a). The modified strain, proposed by Zinoviev [19,20], is the special strain that enables us to consider the effect of deformation in each fiber direction on the damage process.

$$
\begin{aligned}
& \bar{\varepsilon}_{11}=\varepsilon_{11}+\nu_{12} \varepsilon_{22} \\
& \bar{\varepsilon}_{22}=\nu_{21} \varepsilon_{11}+\varepsilon_{22}
\end{aligned}
$$

The modified strain is not applicable in the case of the shear damage parameter $\omega_{12}$, thus $\bar{\varepsilon}_{12}=\gamma_{12}$. To prevent material "healing," the rate of change of all damage parameters must be non-negative.

$$
\dot{\omega} \geqslant 0
$$

Verification analyses are conducted to test the formulation for various plastic recovery factors $\xi$. A saw-tooth displacement amplitude function is assumed, Fig. 9, which consists of three successive unloadings that occur from progressively higher stress values. As shown by Fig. 10, plastic recovery factors of $0.5,0.75$, and 1.0 all provide the desired unloading response.

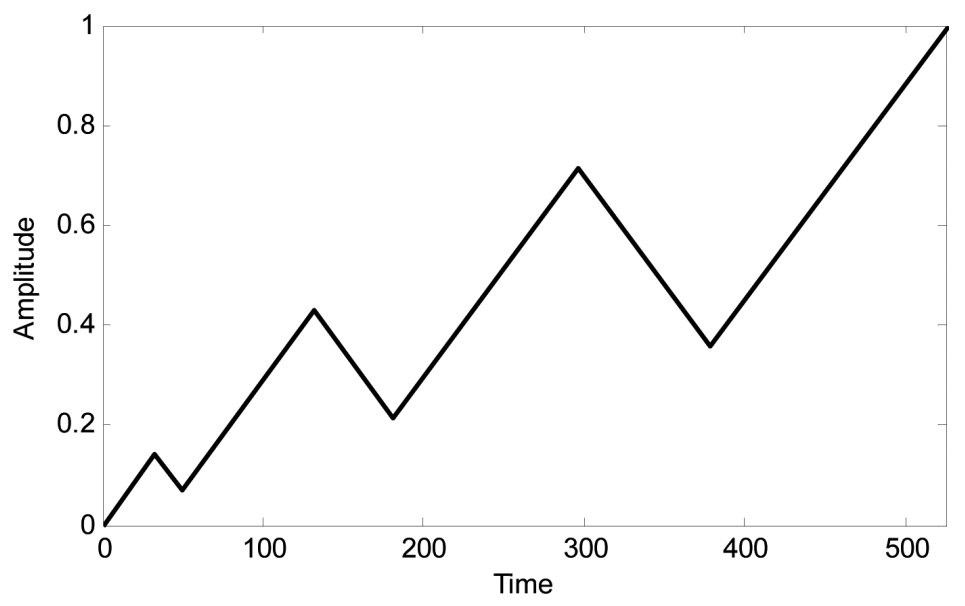

Fig. 9. Input displacement amplitude function.

\section{Experiments}

The shock tube experimental data is based on the work of Baker and Telegadas [12]. The conical shock tube, shown schematically in Fig. 11, is a twenty foot long water-filled tube that is used for shock testing of small items. An explosive charge, positioned at the end of a cone of water, generates a focused shock wavefront similar to free-field underwater explosions. For this test series, an air-backed circular composite specimen is positioned at the opposite end of the tube, and is mounted normal to the shock front on a specially designed slider assembly. The slider assembly allows the fixture to slide backward after the initial wave impingement. As a result, pressure inside the tube dissipates minimizing the effect of the gas bubble. Photographs of the assembly are shown in Figs 12 and 13.

The test instrumentation included three pressure transducers inside the tube (at various distances from the specimen) as well as strain gauges on each face of the composite plate. A schematic of a typical strain gauge layout is shown in Fig. 14. For most of the experiments, there were three strain gauges on the front (wet side) and six on the back (dry side) of the specimen.

Fourteen conical shock tube experiments were performed, consisting of an Aluminum specimen calibration test and thirteen composite specimen tests. All specimens had an overall diameter of 10.375", although the unsupported diameter (once installed in the text fixture) was approximately 7.5". For the composite specimen tests, three different specimen thicknesses were used to induce various levels of damage during the tests. Overall, four 0.86 " thick, five 
(a)

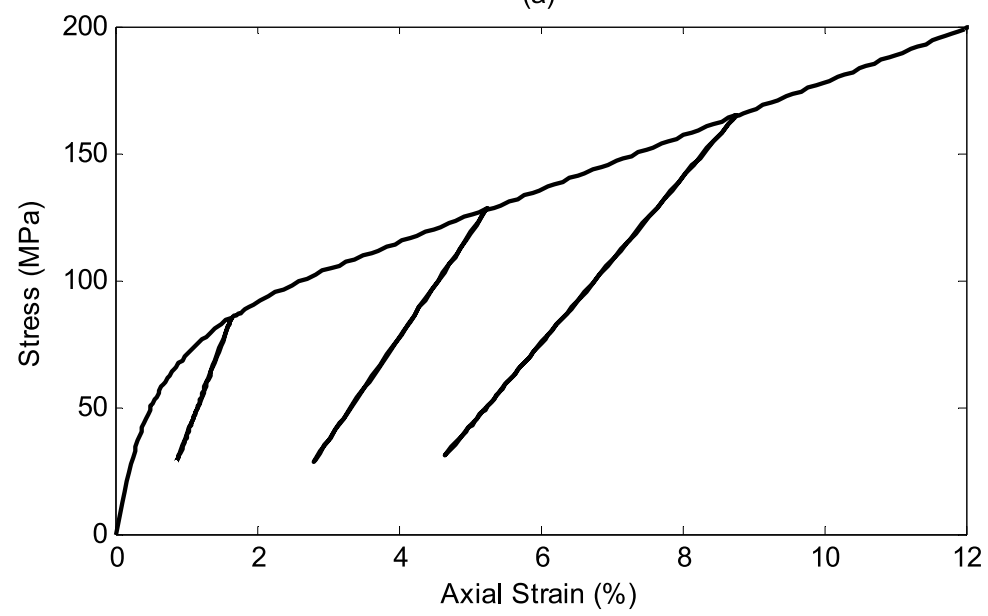

(b)

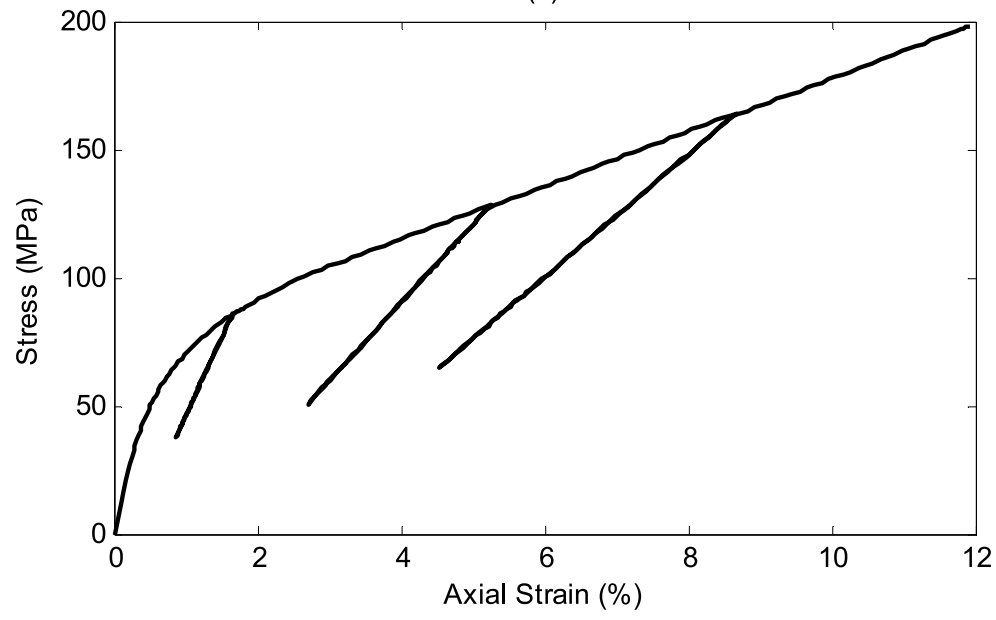

(c)

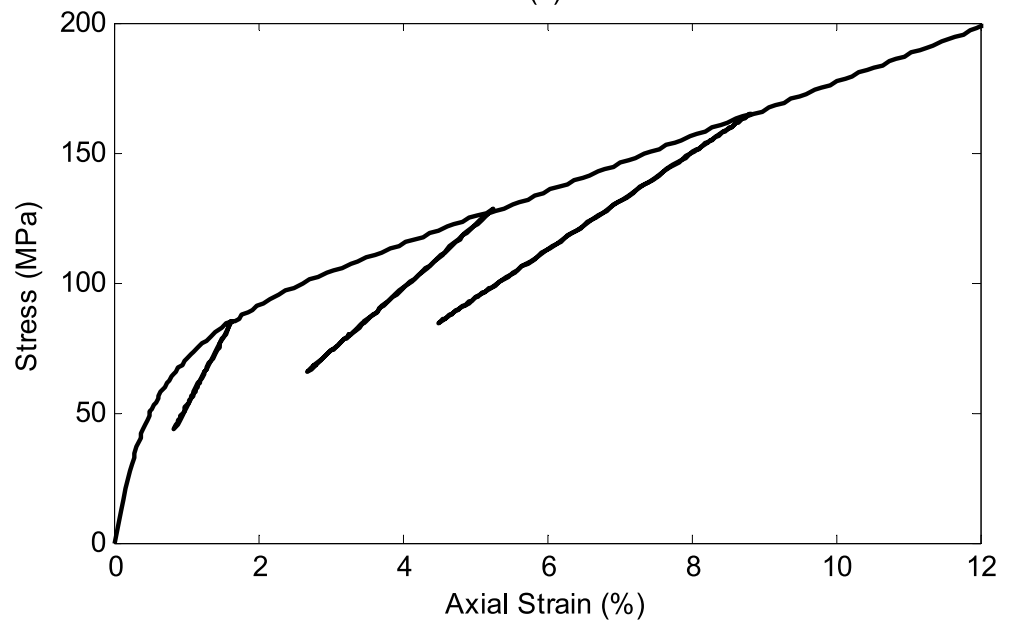

Fig. 10. Verification of unloading constitutive model (a) $\xi=0.5$ (b) $\xi=0.75$ (c) $\xi=1.0$. 


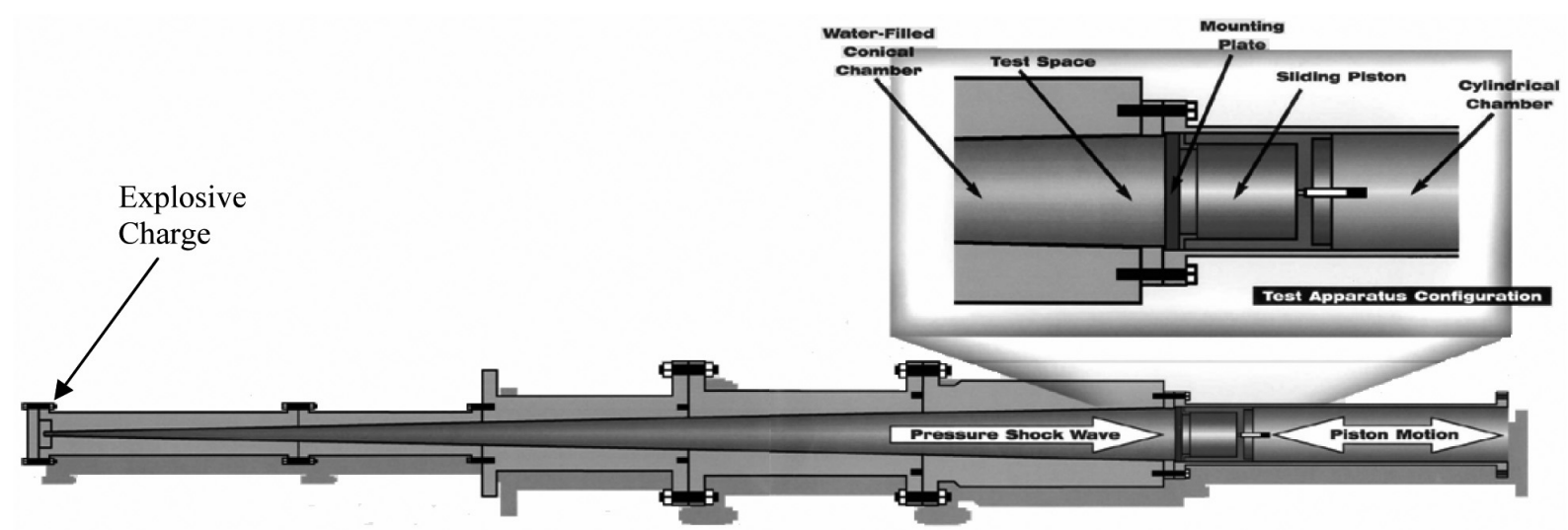

Fig. 11. Schematic of conical shock tube, from Baker and Telegadas [12].

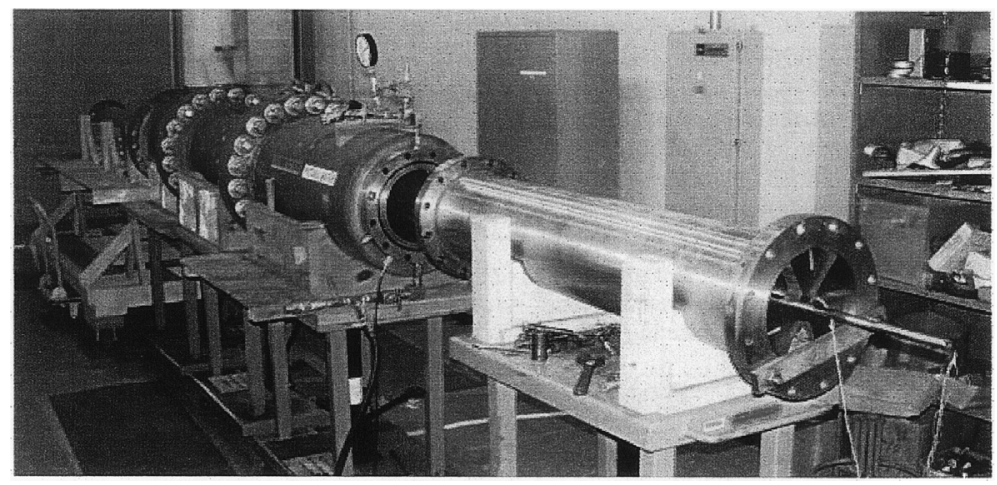

Fig. 12. Conical shock tube, from Baker and Telegadas [12].

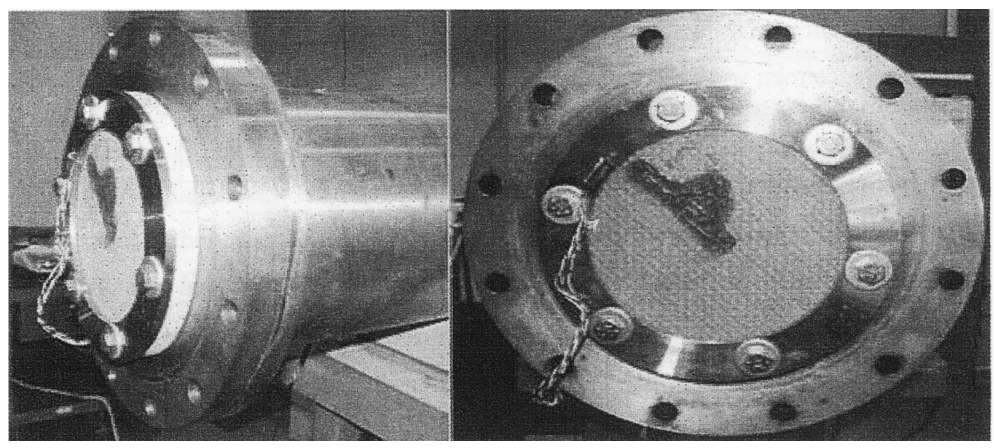

Fig. 13. Slider assembly with installed composite specimen, from Baker and Telegadas [12].

0.58 " thick and four 0.38 " thick specimens were tested. The thickest specimens ( 0.86 ") were designed to remain linear and undamaged during the tests.

After the experiments, non-destructive ultrasound testing was performed to identify any subsurface damage that could not be visually observed. Ultrasound scans confirmed that the 0.86 " thick specimens had no apparent damage. Scans of the 0.58 " specimens indicated internal damage across the entire unsupported mid-plane of the disk. These likely corresponded to delamination. The 0.38 " thick specimens had significant amounts of damage that could be visually observed. This damage involved multiple delaminations through the thickness forming an annular pattern around the edge support, as well as some local fiber failure near the center of the disk. 


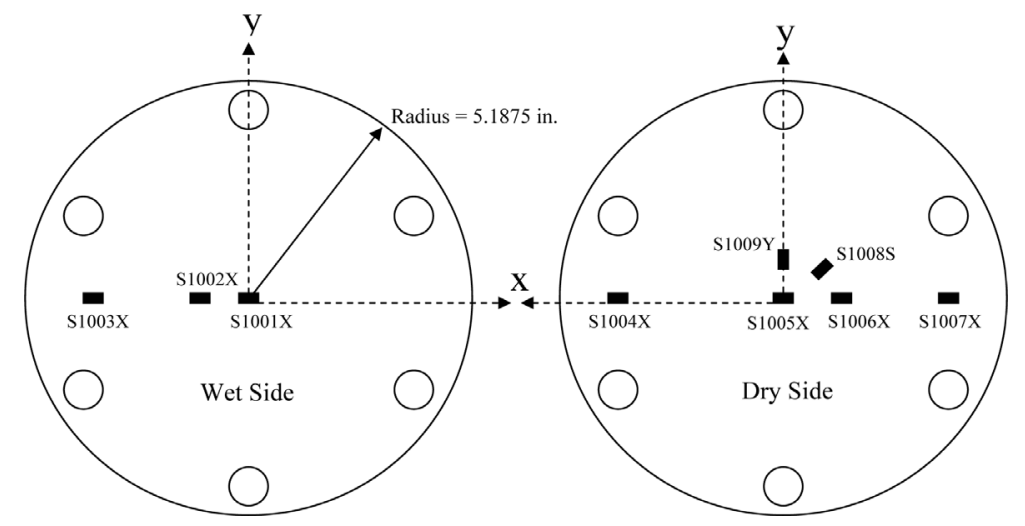

Fig. 14. Typical strain Gauge layout.

\section{Material parameters}

The test specimens are woven E-glass / Vinyl Ester composite with $24 \mathrm{oz} / \mathrm{yd}^{2}$ woven roving (plain weave) fabric. Each laminate is constructed using a symmetric quasi-isotropic stacking sequence. The laminate ply configurations are shown in Table 1, while lamina elastic properties are shown in Table 2.

Table 1

Laminate configurations

\begin{tabular}{ccc}
\hline Nominal thickness (in) & Lay-up & No. Plies \\
\hline 0.38 & {$\left[(0 / 45 / 90 /-45)_{2}\right]_{s}$} & 16 \\
0.58 & {$\left[(0 / 45 / 90 /-45)_{3}\right]_{s}$} & 24 \\
0.86 & {$\left[(0 / 45 / 90 /-45)_{4} / 90 / 45\right]_{s}$} & 36 \\
\hline
\end{tabular}

Table 2

Elastic mechanical properties for woven E-glass / vinyl easter lamina

\begin{tabular}{cccccccc}
\hline $\mathrm{E}_{11}(\mathrm{psi})$ & $\mathrm{E}_{22}(\mathrm{psi})$ & $\mathrm{E}_{33}(\mathrm{psi})$ & $\mathrm{v}_{12}$ & $\mathrm{v}_{13}, \mathrm{v}_{23}$ & $\mathrm{G}_{12}(\mathrm{psi})$ & $\mathrm{G}_{13}(\mathrm{psi})$ & $\mathrm{G}_{23}(\mathrm{psi})$ \\
\hline $3,494,000$ & $3,494,000$ & $1,507,000$ & 0.099 & 0.314 & 560,000 & 545,000 & 545,000 \\
\hline
\end{tabular}

The elastic properties shown in Table 2 are input into the non-linear model. Since only the lamina properties in Table 2 were available, some assumptions were necessary to develop the non-linear constitutive model parameters required for analysis. First, we invoke a common assumption for woven composite, which is that the behavior in the fiber directions is linear (i.e., vanishing plastic strain). Second, we assume that the matrix is the sole source of material nonlinearity and that all matrix dominated material parameters are derived from a similar material system that also used Vinyl ester resin. The combination of these assumptions implies that $a_{11}=a_{12}=a_{22}=0$, and the plastic potential function of Eq. (1) reduces to:

$$
f\left(\sigma_{i j}\right)=a_{66} \sigma_{12}^{2}
$$

Based on analytical correlations of similar material systems that incorporate the same matrix material [17,18], we take $a_{66}=20$. Noting the relationship between the effective stress and the potential function, we obtain:

$$
\bar{\sigma}=\sqrt{3 f} \approx 7.7 \cdot \sigma_{12}
$$

Material non-linearity is then based on the power law relationship between effective stress and effective plastic strain (Eq. (3)). This equation is displayed graphically in Fig. 15. 


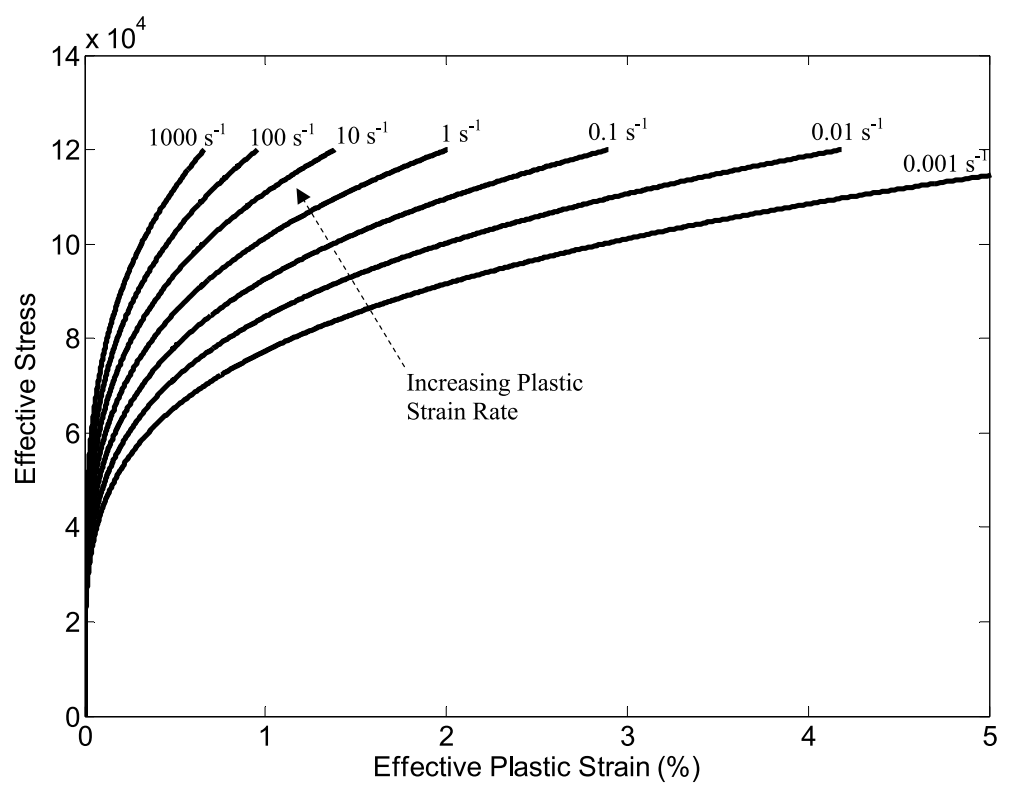

Fig. 15. Master curves for various effective plastic strain rates.

\section{Simulation}

Finite element models of the conical shock tube tests are developed for analysis with Abaqus. The conical shock tube is a complex fixture that requires consideration of many unique aspects of the physical behavior. The most significant aspects included characterization of the shock wave, non-linear fluid modeling, and modeling the specimen edge support.

The incident pressure wave is derived using pressure data recorded by transducers inside the tube. The test data is not used directly since it also includes the reflected and radiated waves from the specimen. By examining the pressure gauge furthest from the specimen (about 32" away) it is possible to clearly observe the incident pressure wave for a brief time period before the arrival of the first reflected wave. The incident pressure wave must be characterized in order to specify the initial conditions of the simulation. The incident pressure wave is represented with a decaying exponential function in the form:

$$
P(t)=P_{0} e^{\frac{-\left(t-t_{0}\right)}{\theta}}
$$

The transient incident pressure amplitude $P(\mathrm{t})$ is defined by a peak pressure $P_{0}$ of 3300 psi and a decay time $\theta$ of 0.2835 milliseconds. A linear curve with a rise time of 0.0165 milliseconds is also used to define the initial pressure increase. Since the pressure is experimentally determined at a point located 32 inches in front of the specimen, approximately 32" of fluid is represented in the finite element model. The transient fluid pressure is initialized at the fluid boundary (32" away from the specimen) based on Eq. (23) above. Figure 16 shows a comparison of the pressure time histories at this location. The simulated incident pressure, as defined using Eq. (23), shows excellent agreement with the measured pressures.

The finite element model (FEM) of the conical shock tube test is shown in Figs 17 and 18. The element types, loads, boundary conditions, and interactions are as noted. The model includes a portion of the fluid, the specimen, and the necessary components of the mounting fixture (retaining ring, fasteners, mounting ring). A half-symmetric model is constructed due to the bolting pattern.

The fluid is represented as a non-linear acoustic medium. Cavitation is included by specifying a pressure limit of zero and disallowing non-physical fluid tension. Initial simulations showed that including cavitation had significant effects on later time response (i.e., after the incident wave passes and reflects off the specimen). Various fluid mesh densities were also tested. The most reasonable predictions of cavitation and fluid structure interaction are obtained by implementing a progressively finer mesh density as the distance from the specimen decreases (See Fig. 17). This 


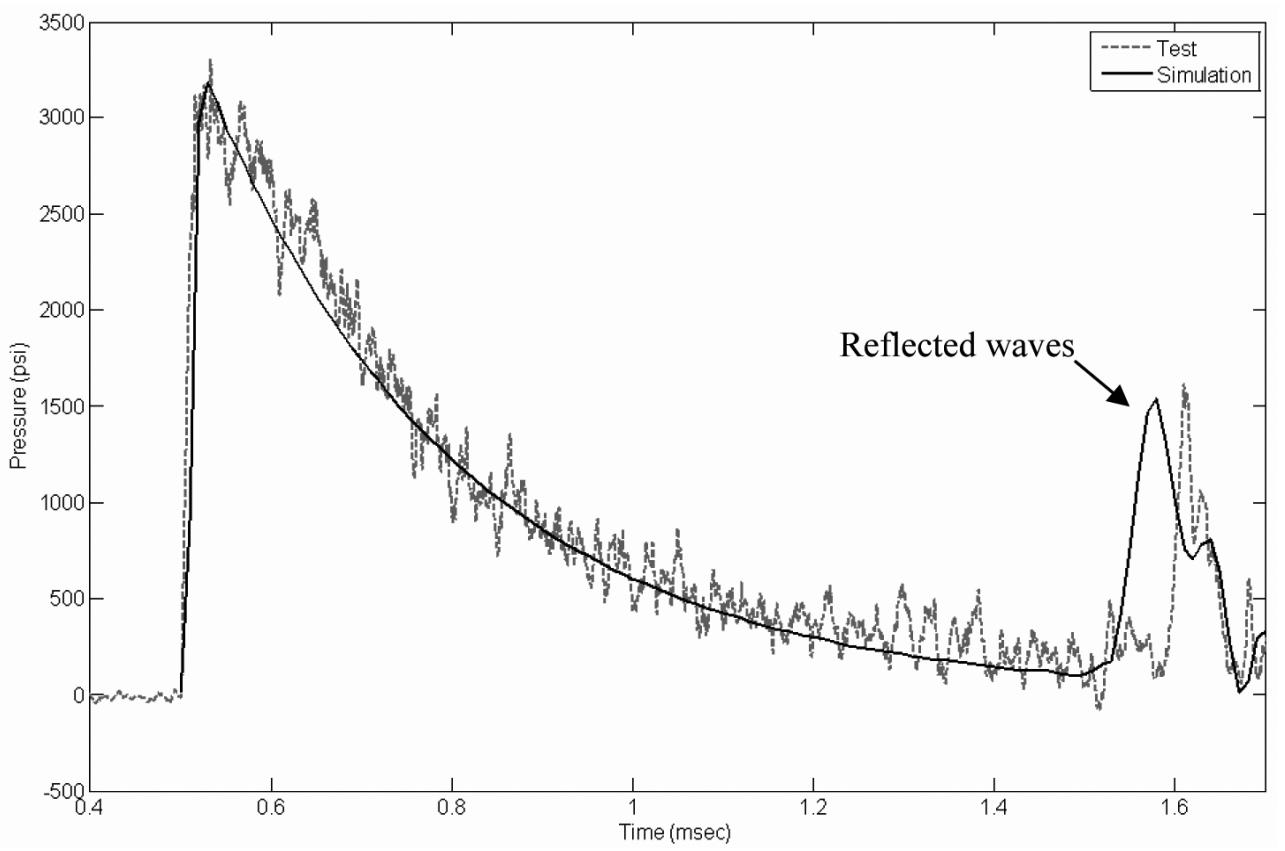

Fig. 16. Comparison of pressure time histories.

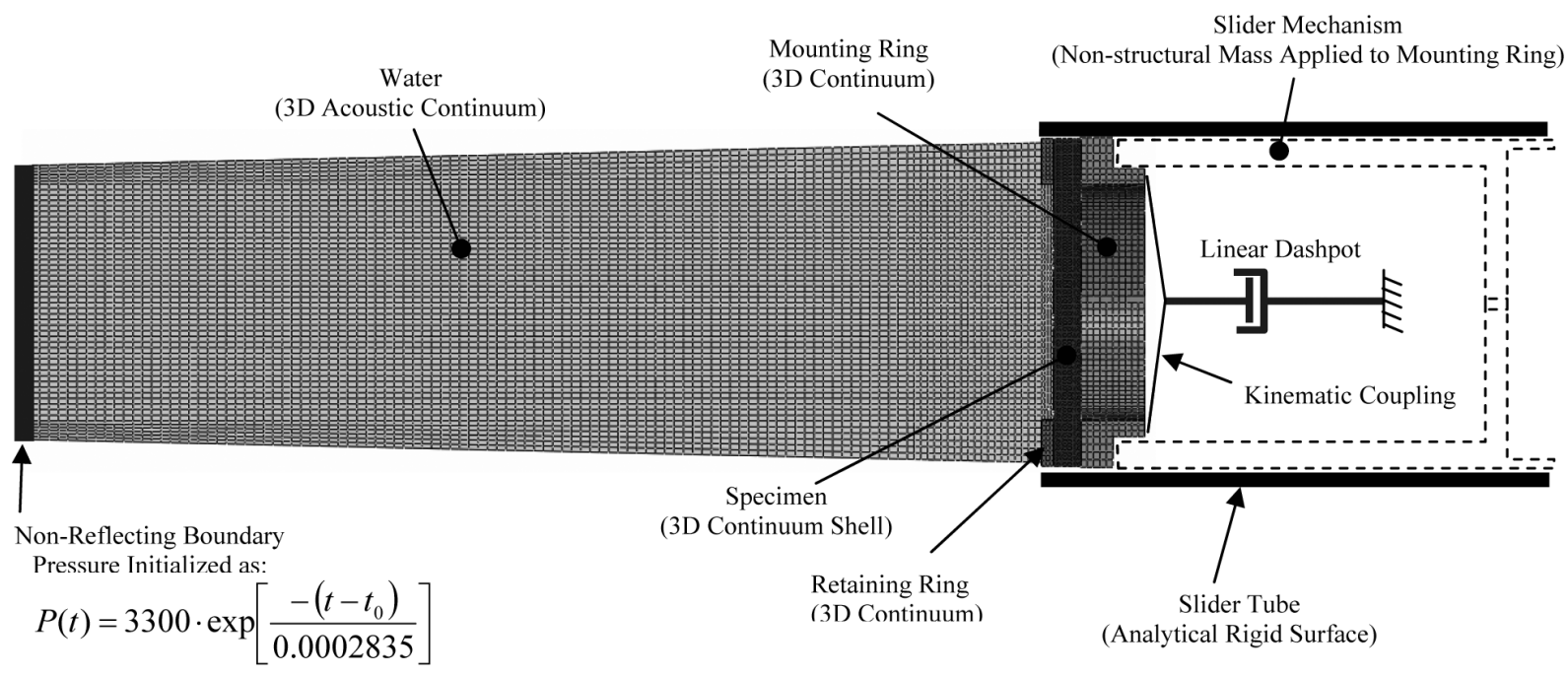

Fig. 17. Finite element model for simulation of conical shock tube test.

is judged by comparing the reflected waves predicted by the simulation to the pressure gauge readings. It is noted, however, that a highly accurate prediction of reflected and radiated waves remains difficult to simulate, and will be somewhat dependent on the fluid element formulations used by different analysis codes.

The specimen is modeled with stacked continuum shell elements (SC8R). The continuum shell is geometrically equivalent to an 8-noded solid element, although mathematically it uses a shell formulation. For a predominantly bending case such as this, the continuum shell provides accurate predictions of strain without requiring a high level of discretization through the thickness (the use of several elements through the thickness was primarily motivated by the details of the laminate cross section definition). The spatial discretization of elements was investigated through several analysis iterations, which showed only a slight change in the strain prediction for finer/coarser meshes. 


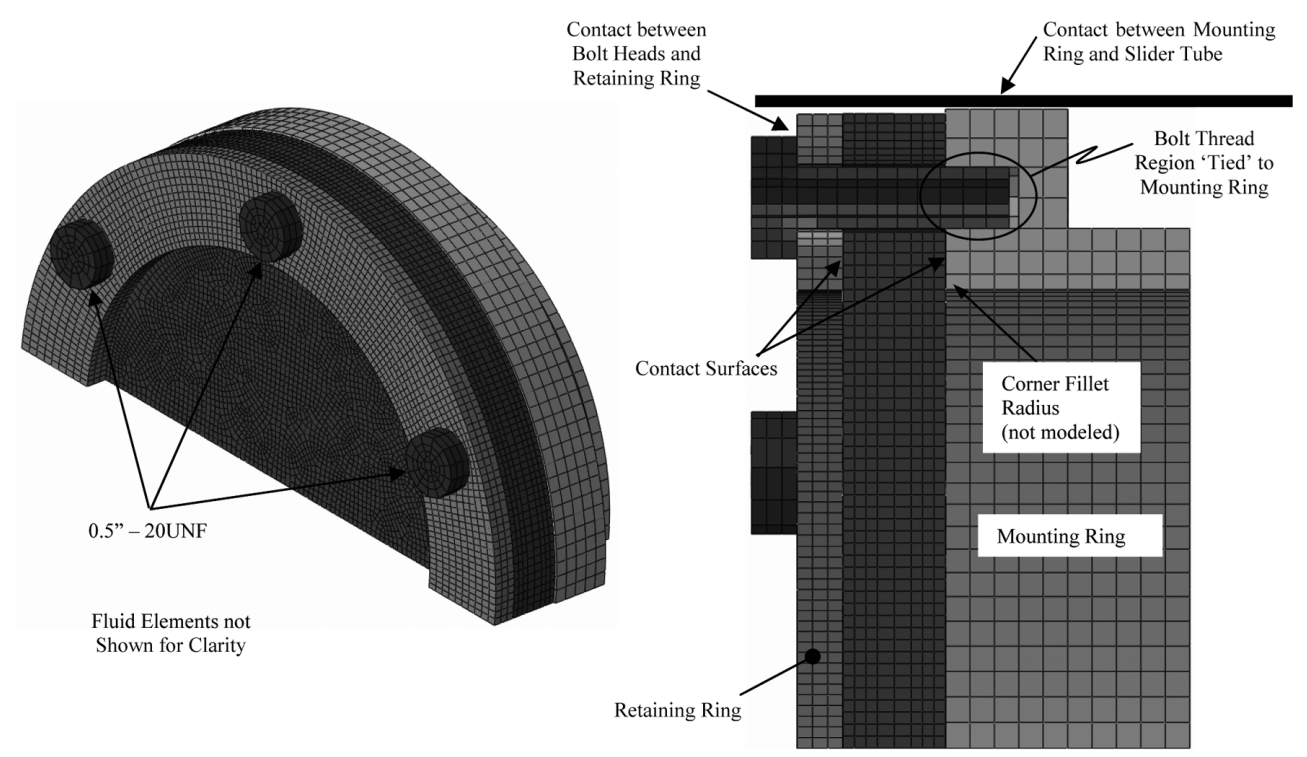

Fig. 18. Finite element model, isometric \& section views.

Modeling the specimen edge support proved challenging since the edge is not perfectly clamped. The specimen is held in place with a 0.375 " steel retaining ring and six $1 / 2$ " diameter bolts. Therefore, the rotation angle at the edge is not zero and the specimen can slide radially after overcoming some unknown frictional resistance. Since no simple boundary condition could represent the physical problem, the edge connection is modeled in detail. Bolts are explicitly modeled with their specified pre-load. To include the effects of fastener pre-load, a temperature change is applied to the bolt shanks. The applied $\Delta T$ is iteratively adjusted to obtain the requisite preload of approximately 25 ksi. Contact surfaces are also included with an estimated dynamic friction coefficient of 0.1. Contact constraints are enforced using the penalty method with the typical master-slave type surface definition. Due to the finer mesh density, the specimen surfaces are defined as the slave surfaces on both the retaining ring and mounting ring interfaces. The retaining ring and mounting ring are represented with solid continuum elements (C3D8). These items are not of specific interest and somewhat coarser mesh densities are used. The solution is fairly insensitive to mesh densities within the retaining ring and mounting ring since both are specified as 'master' surfaces in the contact algorithm. As such, nodes associated with the specimen are not allowed to penetrate any of the surface facets.

As noted in Fig. 18, the mounting ring contains a slight fillet radius in way of the specimen that is approximated as a square corner. The purpose of the fillet radius is to prevent a sharp edge on the mounting ring from scoring the specimen during the test. To compensate for the fact the fillet is not represented, the corresponding inner ring of element faces are excluded from the contact definition. Computationally, this is most reasonable since no contact would be expected in way of the fillet and including this exact geometry would have required some considerably smaller elements. Recall that the time step size in an explicit dynamics procedure is controlled by the smallest element length. Thus, attempting to model the fillet would have unnecessarily exacerbated the computations.

The effects of stiffness proportional damping are also investigated. The addition of a high frequency damping provides a better conditioned response, however, the stable time step size is substantially decreased. For comparison purposes, all simulations shown herein are executed without damping. However, it is recognized that some damping level is physically real and higher frequency response predictions are likely overstated.

\section{Results and discussion}

The fluid pressures predicted by the simulation are investigated first. This provided a means to understand the specimen response, and also allowed verification of the pressure wave definition. As shown by Fig. 19 (frames 1 and $2)$, the wave is correctly initialized such that the peak pressure $(\sim 3300 \mathrm{psi})$ corresponds to test measurements. As 


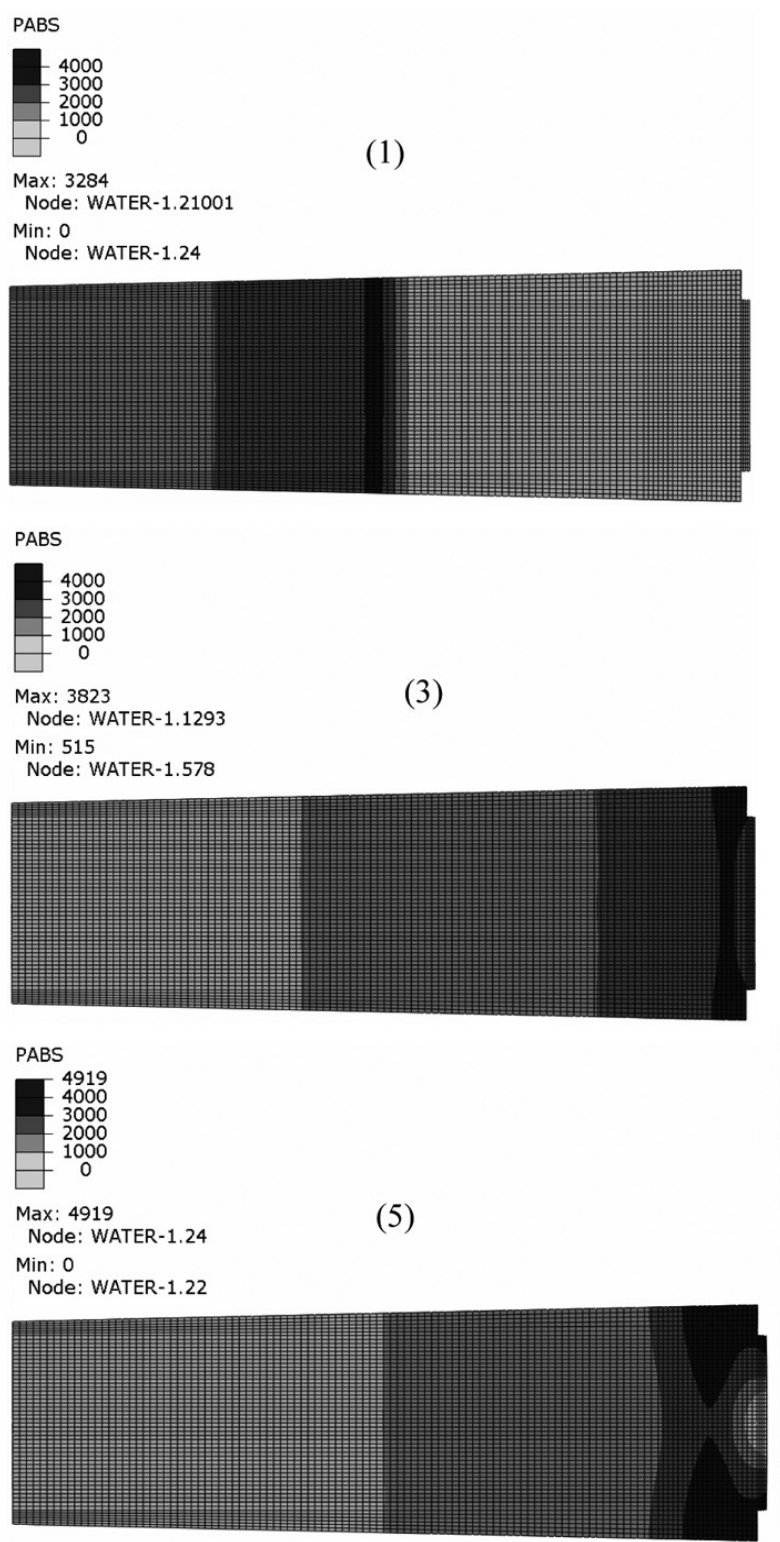

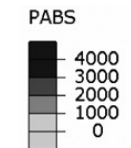

Max: 3052

Node: WATER-1.27384

Min: 0

Node: WATER-1.1385

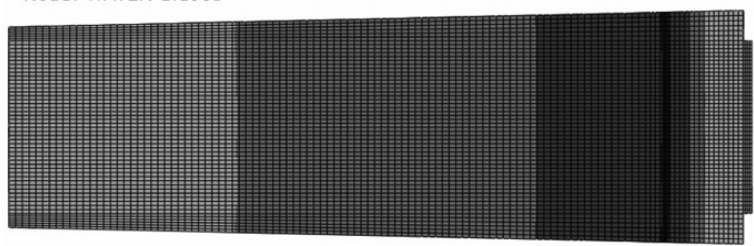

PABS

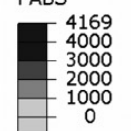

Max: 4169

Node: WATER-1.27923

Min: 462

Node: WATER-1.13

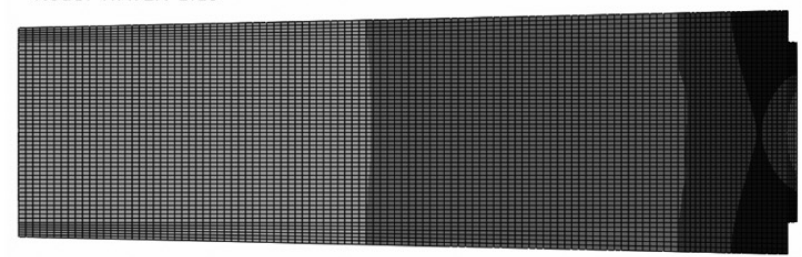

PABS

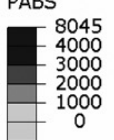

Max: 8045

Node: WATER-1.27620

Min: 0

Node: WATER-1.22

(2)

(4)

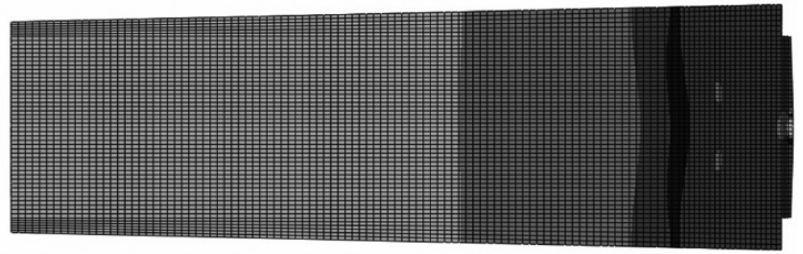

Fig. 19. Simulated fluid pressures during incident wave impingement.

the wave hits the reflecting boundaries of the fixture and specimen, there is a well defined region of higher pressure around the edge of the fixture and specimen (frames 3 and 4). As the specimen begins to deform, the fluid cavitates near the center of the disk (frame5). As the incident wave interacts with the specimen, reflected and radiated waves are transmitted back down the tube (frame 6).

As a result of the non-uniform pressure loading, which involves higher pressures near the edge and lower pressures near the center, the specimen exhibits a noticeable mode II vibration. This was somewhat unexpected, and was not fully understood until detailed fluid structure interaction simulations were performed and visualized. The local increase in pressure forms as a result of (1) the impedance mismatch between the fixture and the composite specimen, and (2) the step created in the flow path by the retaining ring causing the wave to reflect off this metal surface just prior to interacting with the specimen. Based on the simulations, pressure magnitudes locally increase to over 4000 psi around the edge of the specimen, while the peak pressure from the incident wave is only 3000 psi. Due to the 
clamped boundary, the edge will experience the highest transverse shear stresses. However, this transverse shear stress is further exaggerated due to the pressure distribution. Based solely on the simulated fluid pressures, the failure process (for 0.58 " and 0.38 " specimens) likely involved an initial delamination near the edge support (at the laminate mid-plane, as a result of high transverse shear stresses) followed by progressive delamination beginning at the edge and propagating toward the center.

The deflections predicted by the simulation are investigated next. Animations of the specimen deformations allow verification of the behavior of edge support (i.e., contact surface interactions). This visualization process also reveals the mode II vibration response, which is easily noticeable in the undamped simulations. The maximum deflections for the 0.58 " specimen are shown in Fig. 20. Maximum deflections at the disk center are approximately 0.37 " for the 0.58 " thick specimen and approximately 0.23 " for the 0.86 " thick specimen. Again, it must be noted that the corner fillet radius of the mounting ring is approximated as a square corner. The corresponding elements are excluded from the contact surface definition, which results in some elements deforming though each other. This effect appears more exaggerated during visualizations with higher magnification factors.
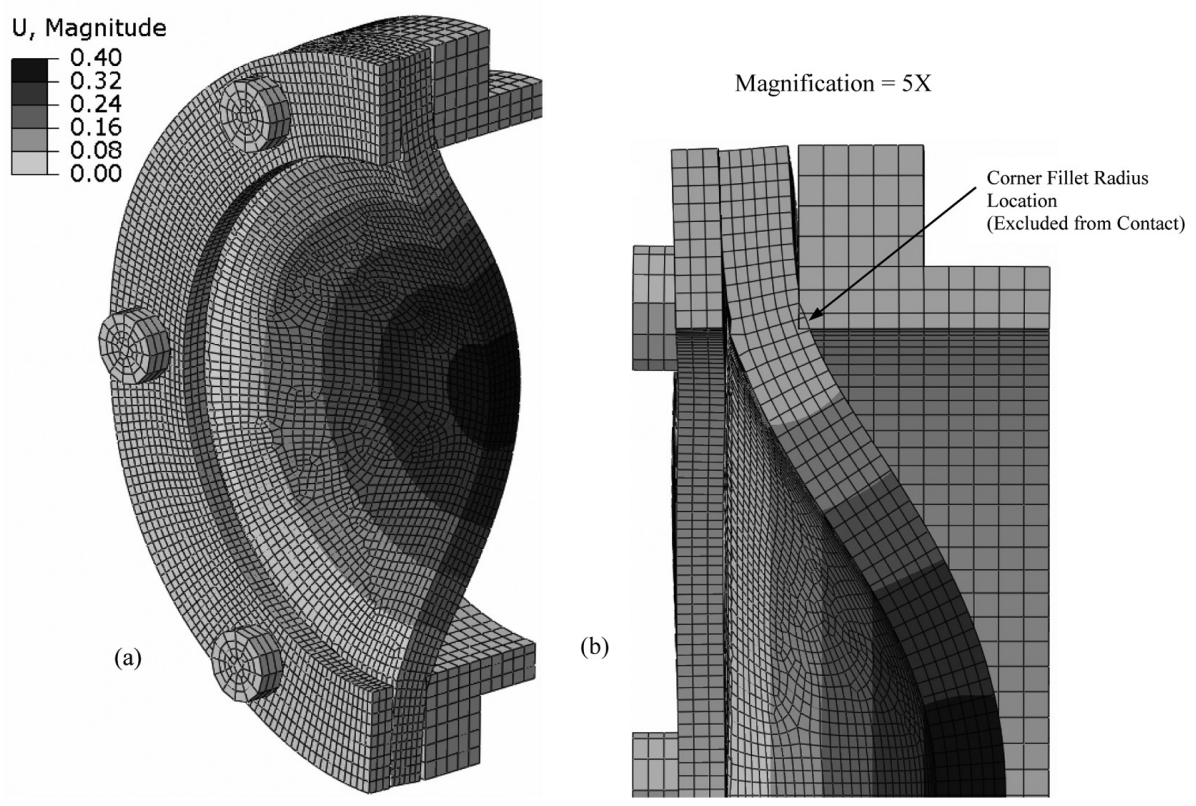

Fig. 20. 0.58" Specimen maximum deformation (a) Isometric view (b) Cross section view.

Finally, comparisons of the calculated and measured strains at various gauge locations are made. Due to excessive gauge failures on the 0.38 " thick specimens, the comparisons focus mainly on the 0.86 " and 0.58 " specimens. Strain correlations are shown in Fig. 21 for the 0.86" thick specimens and Fig. 22 for the 0.58 " specimens. To provide a quantitative measure of the degree of correlation, the Russell comprehensive error factors are calculated. The Russell comprehensive error [21] is an unbiased error measure that considers both magnitude and phase error between two transient signals. Based on Russell's recommended guidelines, an error factor below 0.28 is considered 'acceptable' correlation, while an error factor below 0.15 is considered 'excellent' correlation. All calculated error factors meet the guidelines for acceptable correlation with the majority less than 0.15 (i.e., excellent correlation). In general, simulations show good agreement for the initial strain rise and fall due to pressure wave impingement. After the initial response, the simulation shows a decrease in correlation. However, this is likely related to the pressure characterization inside the tube (e.g., cavitation modeling) and is not directly influenced by the material model.

Peak strains are consistently over-predicted by the simulation. The average percentage difference is approximately $25 \%$. The main factors contributing to the discrepancy are the representation of the specimen boundary conditions, the lack of structural damping in the analysis, and the application of 2D planar theory to a case where through-thickness stresses are not negligible. It is also noted that for 0.58 " specimens the center delaminations likely relieved a portion 

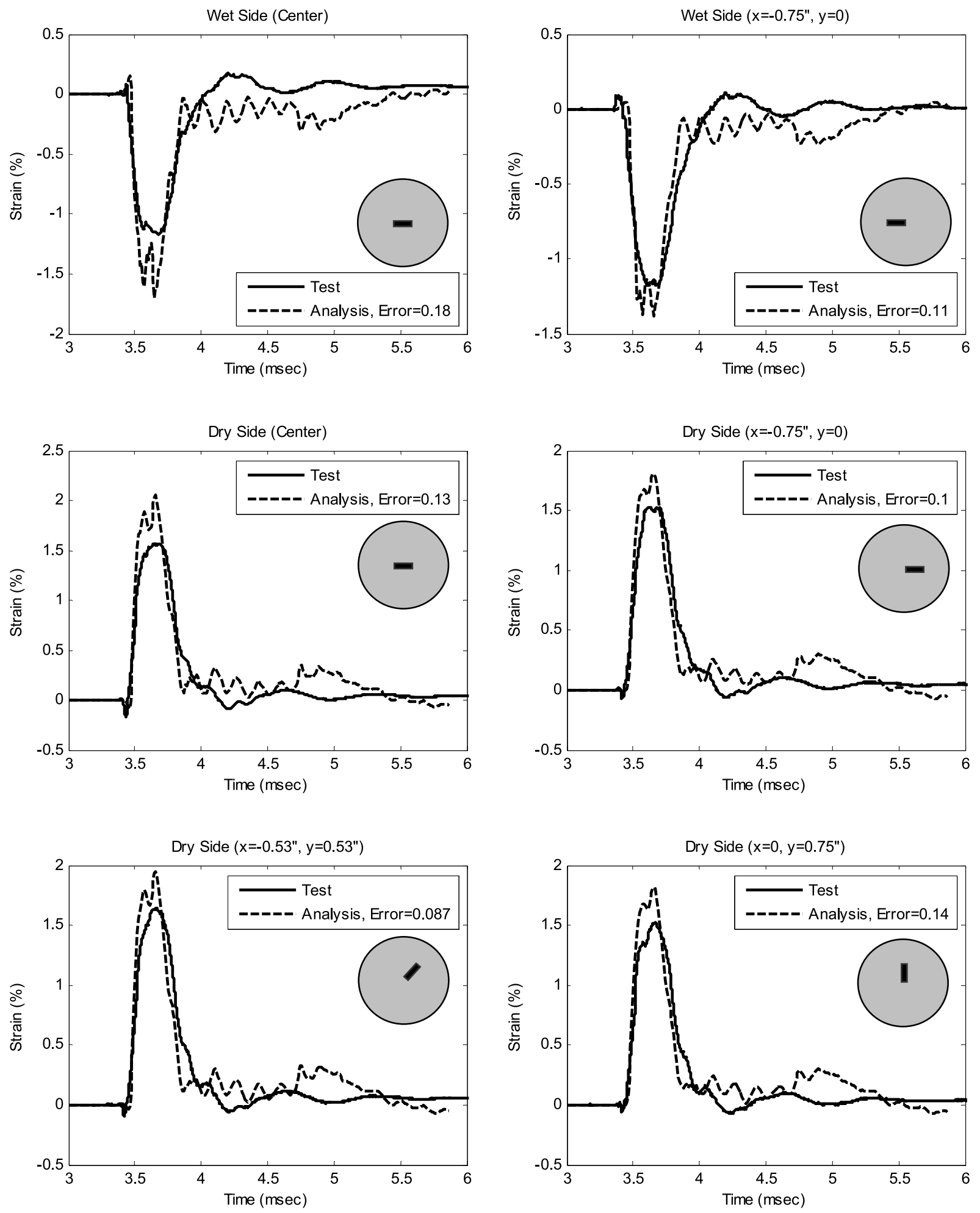

Fig. 21. Strain vs. time for $0.86 "$ thick specimen. 

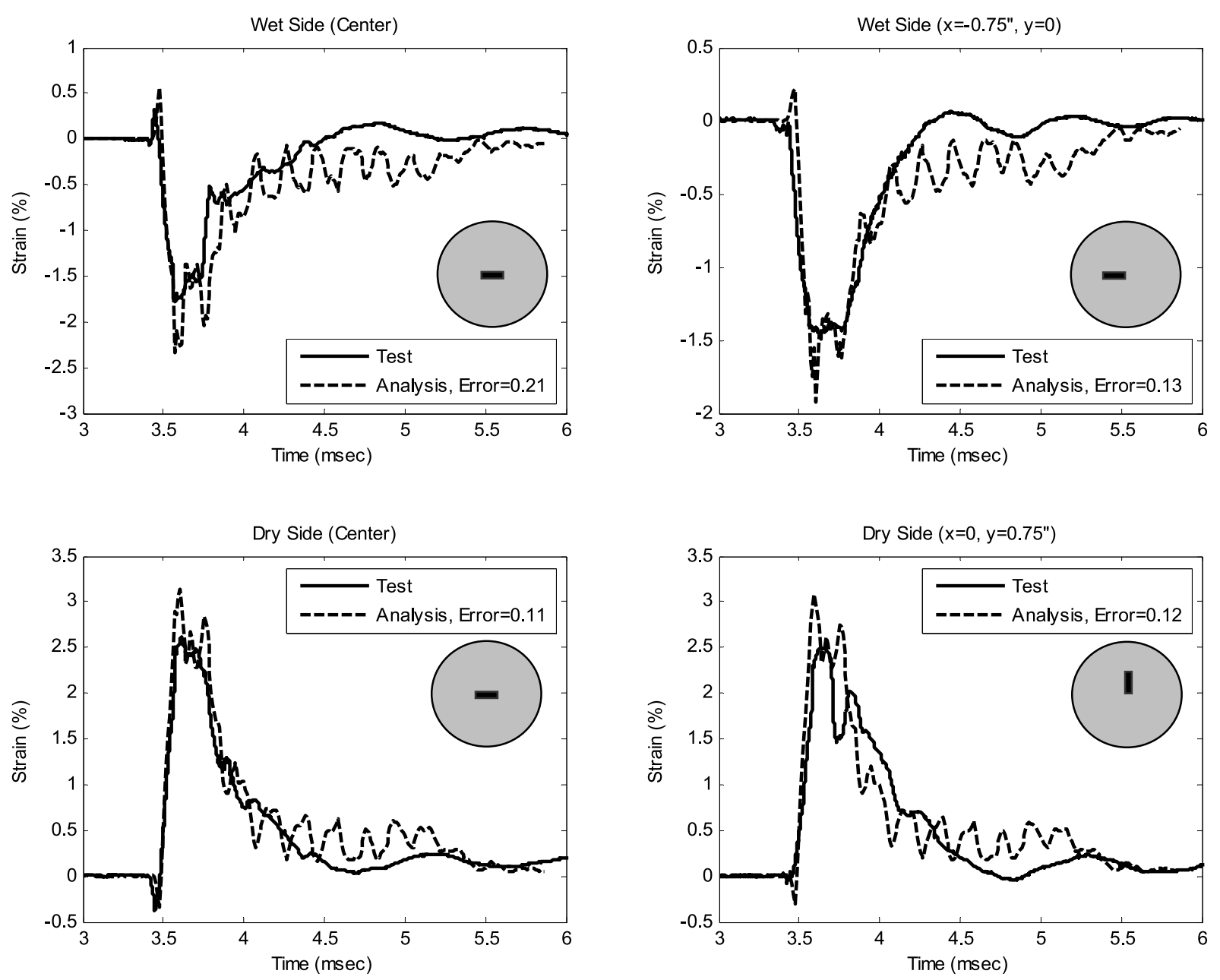

Fig. 22. Strain vs. time for $0.58 "$ thick specimen.

of the strain at surface gauge locations. Considering the limited material data that was available to calibrate the model, the predictions are judged satisfactory. However, it is recommended that other experiments also be considered to support a more complete validation of the theory. These should involve cases where material non-linearity is more significant and higher strains (without simultaneous delaminations) can be achieved (and measured).

The non-linear material model is also able to provide a quantitative measure of ply damage in the form of an internal state variable (i.e., damage parameter). The model provides three damage parameters. These correspond to damage perpendicular to the laminate warp direction, damage perpendicular to the fill direction, and damage in shear. The damage parameters are monotonically increasing state variables. A value of 0 corresponds to the undamaged (virgin) material, while a value of 1 indicates complete damage (i.e., zero stiffness). Because we have assumed linear behavior in fiber directions, the shear stress-strain response is the sole source of nonlinearity. Consequently, the only non-zero damage parameter is the shear damage $\left(\omega_{12}\right)$.

Figure 23 shows the shear damage parameter for the 0.58 " THK specimen. Results for four different plies (each with a different orientation) are shown. As expected, the level of damage decreases with decreasing distance from the laminate mid-plane. In addition, the damage is highest along the outer diameter of the disk, with little to no damage occurring near the center. This is the case since the edge is clamped and the maximum plate bending moment is the moment about the circumferential axis $\left(M_{\theta}\right)$ near the outer edge.

Spatially, we observe that the shear damage increases as the angle between the applied stress and the reinforcement direction approaches $45^{\circ}$. In the circular disk, the applied stress consistently acts in the radial direction. This 

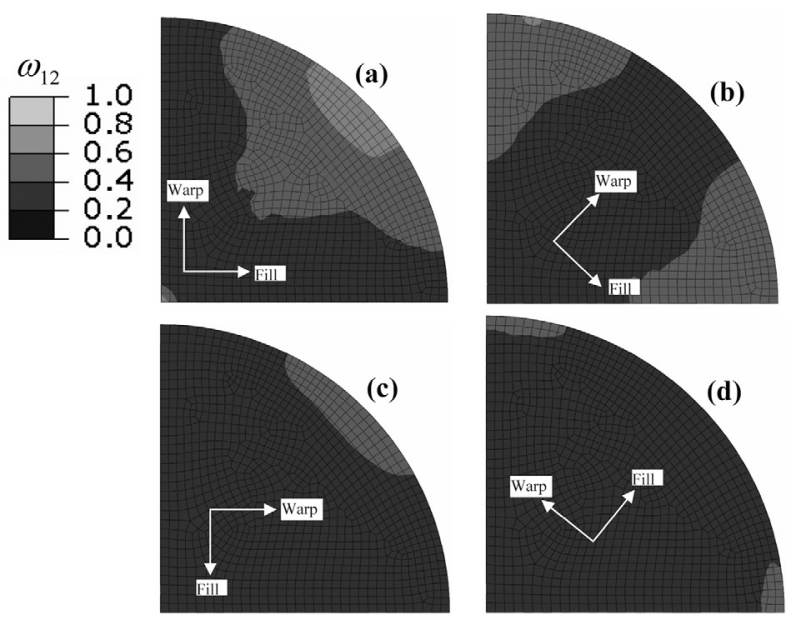

Fig. 23. Shear damage parameter for $0.58^{\prime \prime}$ THK specimen (a) Dry side, 1 st ply, $0^{\circ}$ orientation (b) Dry side, 2 nd ply, $45^{\circ}$ orientation (c) Dry side, 3rd ply, $90^{\circ}$ orientation (d) Dry side, 4 th ply, $-45^{\circ}$ orientation.

provides the explanation for the variation of the shear damage parameter in Fig. 23. For example, in Fig. 23(a), the ply orientation is $0^{\circ}$, giving the maximum damage at $45^{\circ}$ clockwise from top dead center. In Fig. 23(b), the ply orientation is $+45^{\circ}$, giving the maximum damage at $0^{\circ}$ and $90^{\circ}$ from top dead center.

\section{Conclusions}

Constitutive theories for loading and unloading responses have been developed and integrated to form a complete theory for transient analyses of woven composite structures subjected to shock loads. Damage is characterized, tracked, and allowed to influence the unloading response. Material unloading is characterized by a single scalar parameter that can be modified for a particular application. The combined theory has been numerically implemented in the Abaqus finite element code to facilitate design application, solution of more complex problems, or simulation of other validation tests. The finite element implementation is accomplished through user material subroutines (UMAT, VUMAT), which define the constitutive theory and progressive failure methodology.

Advanced simulation techniques can be used to model the behavior of composite structures subjected to underwater shock loading. The conical shock tube is an effective test method to investigate high-rate dynamic loading with fluid structure interaction. The data is useful to verify and compare existing material models, as well as evaluate new material models. In this study, the simulation results were in reasonably good agreement with experimental data.

\section{References}

[1] L. Xing, Progressive Failure of Large Deformation Woven Composites under Tensile Loading, PhD Dissertation, University of Connecticut, Department of Mechanical Engineering, 2007.

[2] K.L. Reifsnider and L. Xing, Large-Deformation Constitutive Theories for Structural Composites: Rate-Dependent Concepts and Effect of Microstructure, Strain 44(1) (2007), 119-125.

[3] L. Xing, K.L. Reifsnider and X. Huang, Progressive Damage Modeling for Large Deformation Loading of Composite Structures, Composites Science and Technology 69 (2009), 780-784.

[4] G. Odegard, K. Searles and M. Kumosa, Nonlinear Analysis of Woven Fabric-reinforced Graphite/PMR-15 Composites under Sheardominated Biaxial Loads, Mechanics of Composite Materials and Structures 7 (2000), 129-152.

[5] S. Ogihara and K.L. Reifsnider, Characterization of Nonlinear Behavior in Woven Composite Laminates, Applied Composite Materials 9 (2002), 249-263.

[6] L.M. Kachanov, Introduction to Continuum Damage Mechanics, Martinus Nijhoff, Boston, 1986

[7] J.W. Lee, D.H. Allen and C.E. Harris, Internal State Variable Approach for Predicting Stiffness Reductions in Fibrous Laminated Composites with Matrix Cracks, Journal of Composite Materials 23 (1989), 1273-1291. 
[8] A. Matzenmiller, J. Lubliner and R.L. Taylor, A Constitutive Model for Anisotropic Damage in Fiber-Composites, Mechanics of Materials 20 (1995), 125-152.

[9] P. Maimi, P.P. Camanho, J.A. Mayugo and C.G. Davila, A Continuum Damage Model for Composite Laminates: Part I - Constitutive Model, Mechanics of Materials 39 (2007), 897-908.

[10] P. Maimi, P.P. Camanho, J.A. Mayugo and C.G. Davila, A Continuum Damage Model for Composite Laminates: Part II - Computational Implementation and Validation, Mechanics of Materials 39 (2007), 909-919.

[11] I. Lapczyk and J.A. Hurtado, Progressive Damage Modeling in Fiber-reinforced Materials, Composites Part A 38 (2007), $2333-2341$.

[12] J.E. Baker and H.K. Telegadas, Conical Shock Tube (CST) Test Report for Composite Plate Specimens, NSWCCD-65-TR-2005/08, 2005.

[13] K.L. Reifsnider and S.W. Case, Damage Tolerance and Durability of Material Systems, John Wiley and Sons, New York, 2002.

[14] C.T. Sun and J.L. Chen, A Simple Flow Rule for Characterizing Nonlinear Behavior of Fiber Composites, Journal of Composite Materials 23 (1989), 1009-1020.

[15] C.A. Weeks and C.T. Sun, Modeling Non-linear Rate-Dependent Behavior in Fiber-Reinforced Composites, Composites Science and Technology 58 (1998), 603-611.

[16] S.V. Thiruppukuzhi and C.T. Sun, Models for the Strain-Rate-Dependent Behavior of Polymer Composites, Composites Science and Technology 61 (2001), 1-12.

[17] D.R. Hufner and M.L. Accorsi, A Progressive Failure Theory for Woven Polymer-Based Composites Subjected to Dynamic Loading, Composite Structures 89(2) (2009), 177-185.

[18] D.R. Hufner, Progressive Failure of Woven Polymer-Based Composites under Dynamic Loading; Theory and Analytical Simulation, PhD Dissertation, University of Connecticut, Department of Civil and Environmental Engineering, 2008.

[19] P.A. Zinoviev, S.V. Grigoriev, O.V. Lebedeva and L.P. Tairova, The Strength of Multilayered Composites Under a Plane-Stress State, Composites Science and Technology 58 (1998), 1209-1223.

[20] P.A. Zinoviev, O.V. Lebedeva and L.P. Tairova, A Coupled Analysis of Experimental and Theoretical Results on the Deformation and Failure of Composite Laminates Under a State of Plane Stress, Composites Science and Technology 62 (2002), 1711-1732.

[21] D.M. Russell, Error Measures for Comparing Transient Data, Part I: Development of a Comprehensive Error Measure, Part II: Error Measures Case Study, in Proceedings of the 68th Shock and Vibration Symposium, November 3-6, 1997. 

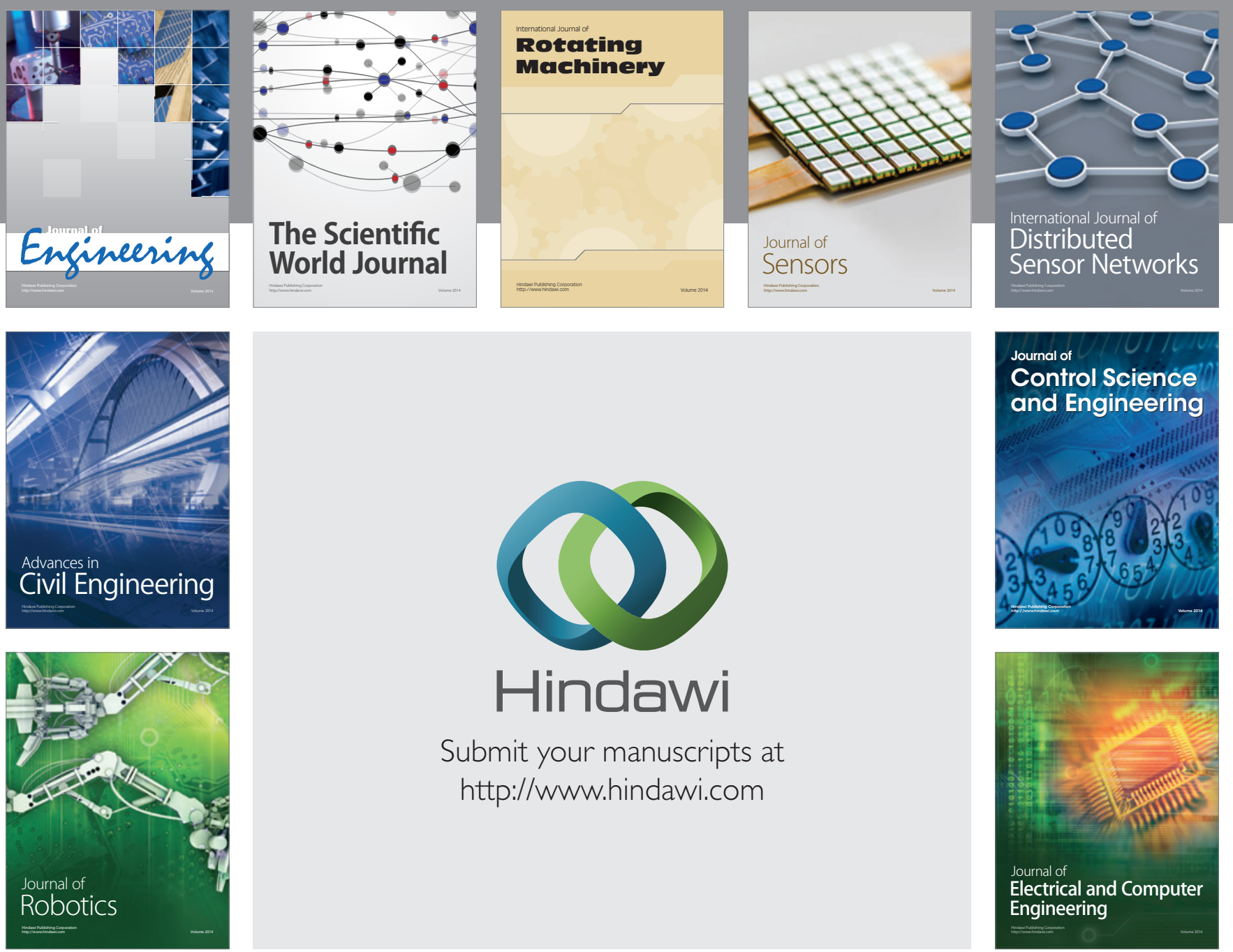

Submit your manuscripts at

http://www.hindawi.com
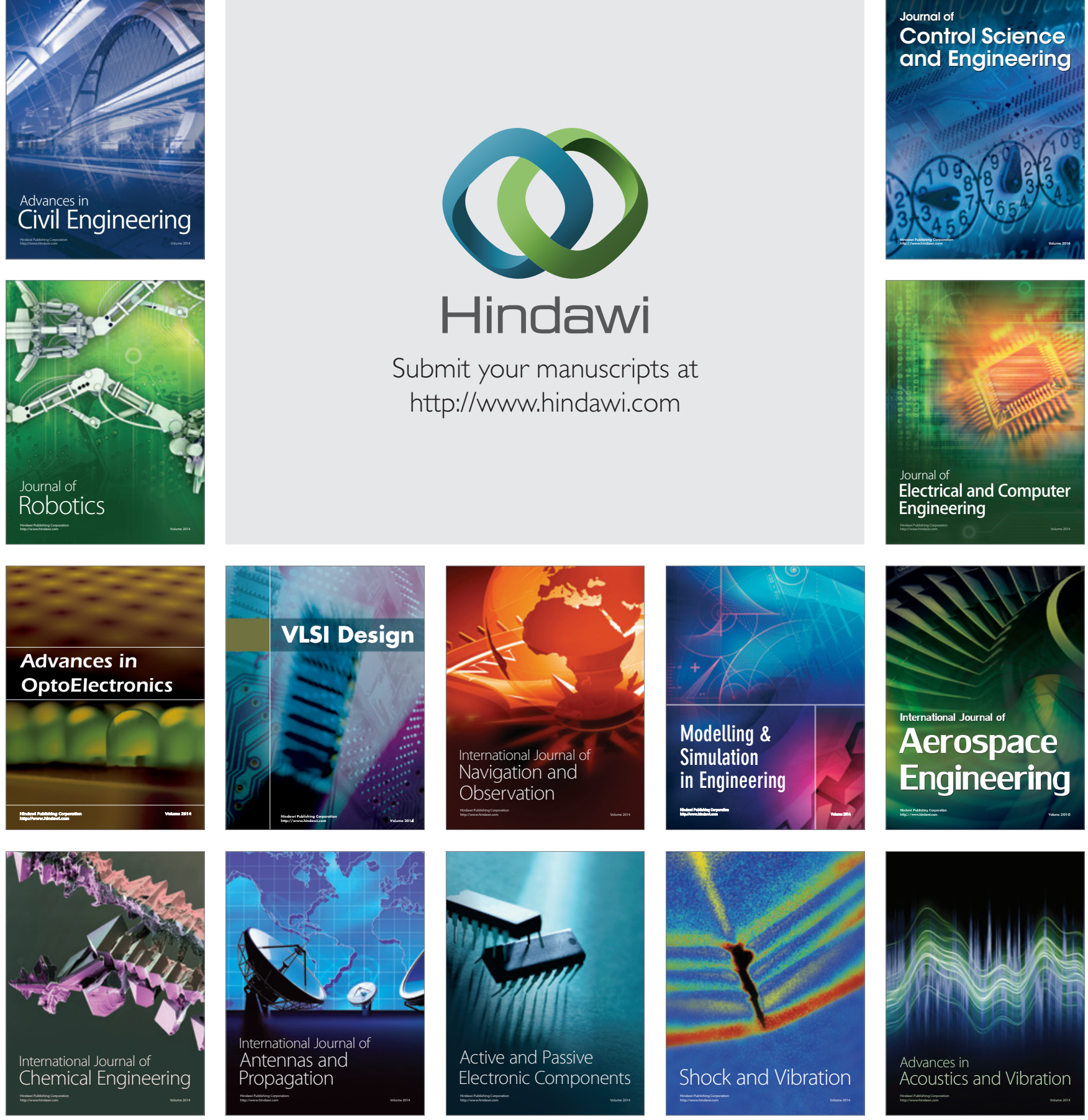